\title{
ASYMPTOTIC PROPERTIES OF POWER VARIATIONS OF LÉVY PROCESSES
}

\author{
JEAN JACOD ${ }^{1}$
}

\begin{abstract}
We determine the asymptotic behavior of the realized power variations, and more generally of sums of a given function $f$ evaluated at the increments of a Lévy process between the successive times $i \Delta_{n}$ for $i=0,1, \ldots, n$. One can elucidate completely the first order behavior, that is the convergence in probability of such sums, possibly after normalization and/or centering: it turns out that there is a rather wide variety of possible behaviors, depending on the structure of jumps and on the chosen test function $f$. As for the associated central limit theorem, one can show some versions of it, but unfortunately in a limited number of cases only: in some other cases a CLT just does not exist.
\end{abstract}

Mathematics Subject Classification. 60F17, 60G51.

Received November 4, 2005. Revised April 4, 2006.

\section{INTRODUCTION}

The behavior of the so-called realized power or multipower variations for discretely observed semimartingales has attracted a lot of attention recently, mainly because of applications in finance: they are used for estimating the volatility or integrated volatility, typically in a stochastic volatility context, with or without jumps. They are useful also when one wants to decide whether a discretely observed process is continuous or has jumps. In all these cases one is looking at what kind of power or multipower or possibly truncated power variations is best for estimating the integrated volatility and/or some functions of the jumps. Let us quote for example [2-4], and some partial results when the process under considerations has jumps may be found in $[5,12]$ or $[13]$, see also [1] in a different context.

Determining the asymptotic behavior of power or multipower variations when the time lag goes to 0 is not a trivial problem, especially when there are jumps. In this paper we consider the simplest possible case: the underlying process $X$ is a Lévy process, and we look at power or truncated power variations only. However, one may hope that the results extend to more general semimartingales, at least for those having "absolutely continuous" characteristics. They also extend to multipower variations, but probably not in a trivial way.

When $X$ is a continuous Lévy process the results are of course elementary, but they become not so simple and indeed quite versatile when there are jumps. However the homogeneity and the independent increments property of $X$ allow to give complete answers for the convergence in probability of (possibly normalized) power variations to a non trivial limit. As for the associated CLT we give a complete answer only when the Gaussian part of $X$ does not vanish, and this probably is the interesting case in practice. When the Gaussian part

\footnotetext{
Keywords and phrases. Central limit theorem, quadratic variation, power variation, Lévy processes.

1 Institut de mathématiques de Jussieu, 175 rue du Chevaleret 75013 Paris, France (CNRS - UMR 7586, and Université Pierre et Marie Curie-P6); jj@ccr.jussieu.fr
} 
vanishes, the existence of a CLT depends on the behavior of the Lévy measure of $X$ near 0, and such a CLT does not necessarily exist: in this paper we essentially do not consider this situation.

Let us be more specific. We have a 1 -dimensional Lévy process $X$ with characteristics $(b, c, F)$ (see Sect. 2 for more details). This process starts at 0 (i.e. $X_{0}=0$ ) and it is sampled at times $\Delta_{n}, 2 \Delta_{n}, \cdots$, where $\Delta_{n}$ is always assumed to go to 0 as $n \rightarrow \infty$. With the notation

$$
\Delta_{i}^{n} Y=Y_{i \Delta_{n}}-Y_{(i-1) \Delta_{n}}
$$

for the increments of any process $Y$, we consider the realized $r$-variation process

$$
\Pi^{n}(r)_{t}:=\sum_{i=1}^{\left[t / \Delta_{n}\right]}\left|\Delta_{i}^{n} X\right|^{r}
$$

where $r \in(0, \infty)$, and also the truncated realized $r$-variation at level $a$ :

$$
\Pi^{n}(r, a)_{t}:=\sum_{i=1}^{\left[t / \Delta_{n}\right]}\left|\Delta_{i}^{n} X\right|^{r} 1_{\left\{\left|\Delta_{i}^{n} X\right| \leq a\right\}} .
$$

We are interested in the asymptotic behavior of $\Pi^{n}(r)$, or of $\Pi^{n}\left(r, a_{n}\right)$ for a sequence of truncation levels $a_{n}$ of the form $a_{n}=a$ or $a_{n}=a \sqrt{\Delta_{n}}$ for some $a>0$, although other choices for $a_{n}$ would also be possible; the choice $a_{n}=a \sqrt{\Delta_{n}}$ is obviously related to the property that $\Delta_{i}^{n} X / \sqrt{\Delta_{n}}$ is $\mathcal{N}(0, c)$ when $X$ is Gaussian, and it reflects the fact that we are mainly interested in the case where $c>0$. The LLN results (that is, the convergence in probability, possibly after normalization) for $\Pi^{n}(r)$ cover all possible situations, but for the truncated version $\Pi^{n}(r, a)$ with a fixed level $a$ there are difficulties due to the non-continuity of the function $x \mapsto|x|^{r} 1_{\{|x| \leq a\}}$.

From a statistical point of view, it is also natural to consider that we sample the process $X$ over the interval $\left[0, T_{n}\right]$, where $T_{n}=n \Delta_{n}$. Then we are interested in the behavior of the variables $\Pi^{n}(r)_{T_{n}}$, or more generally of the processes

$$
\bar{\Pi}^{n}(r)_{t}=\sum_{i=1}^{[n t]}\left|\Delta_{i}^{n} X\right|^{r}, \quad \bar{\Pi}^{n}(r, a)_{t}=\sum_{i=1}^{[n t]}\left|\Delta_{i}^{n} X\right|^{r} 1_{\left\{\left|\Delta_{i}^{n} X\right| \leq a\right\}},
$$

so $\Pi^{n}(r, a)_{T_{n}}=\bar{\Pi}^{n}(r, a)_{1}$. In practice two cases are of interest: one is when $T_{n}=T$ does not depend on $n$ (that is $\Delta_{n}=T / n$ for some $T>0$ ), and in this case $\Pi^{n}(r, a)$ and $\bar{\Pi}^{n}(r, a)$ are essentially identical since $\bar{\Pi}^{n}(r, a)_{t}=\Pi^{n}(r, a)_{t / T}$. The other case is when $T_{n} \rightarrow \infty$ : the results are then much simpler than for $\Pi^{n}(r, a)$, but they require some integrability which holds for the truncated version, but not in general for $\bar{\Pi}^{n}(r)_{t}$.

The two afore-mentioned difficulties - lack of continuity and lack of integrability - lead us to consider slightly more general processes, not really any more difficult to study. More precisely, for any function $f$ on $\mathbb{R}$ we set

$$
V^{n}(f)_{t}:=\sum_{i=1}^{\left[t / \Delta_{n}\right]} f\left(\Delta_{i}^{n} X\right), \quad \bar{V}^{n}(f)_{t}:=\sum_{i=1}^{[n t]} f\left(\Delta_{i}^{n} X\right)
$$

Then $\Pi^{n}(r, a)=V^{n}(f)$ with $f(x)=|x|^{r} 1_{\{|x| \leq a\}}$ (for $\left.a \in(0, \infty]\right)$, and accordingly for $\bar{\Pi}^{n}(r, a)$. The truncated case with a truncation level $a \sqrt{\Delta_{n}}$ is accommodated by looking at the following modified processes:

$$
V^{\prime n}(f)_{t}:=\sum_{i=1}^{\left[t / \Delta_{n}\right]} f\left(\Delta_{i}^{n} X / \sqrt{\Delta_{n}}\right), \quad \bar{V}^{\prime n}(f)_{t}:=\sum_{i=1}^{[n t]} f\left(\Delta_{i}^{n} X / \sqrt{\Delta_{n}}\right) .
$$


So $\Pi^{n}\left(r, a \sqrt{\Delta_{n}}\right)=\Delta_{n}^{r / 2} V^{\prime n}(f)$, with $f(x)=|x|^{r} 1_{\{|x| \leq a\}}$ again.

For the processes $V^{n}(f)$ and $\bar{V}^{n}(f)$, it turns out that the crucial property of $f$ is its behavior near 0 . Depending on the case at hand, we will restrict our attention to functions which, for some $r>0$, are o $\left(|x|^{r}\right)$ or $\mathrm{O}\left(|x|^{r}\right)$ as $x \rightarrow 0$, or even which coincide with the function $x \mapsto|x|^{r}$ on a neighborhood of 0 .

For the processes $V^{\prime n}(f)$ and $\bar{V}^{\prime n}(f)$, however, the whole function $f$ is important and the behavior of $f$ near 0 does not play any specific role. On the other hand the special truncation we consider $\left(\right.$ at $\sqrt{\Delta_{n}}$ ) leads us to give result only when the Gaussian part of $X$ is not vanishing. If it were, other "truncation rates" would be more appropriate.

Let us mention that the results of this paper are connected in some loose way with results about sums of the form $\sum_{i=1}^{n} f_{n}\left(b_{n}^{-1} S_{i}\right)$ for suitable functions $f_{n}$, where $S_{n}$ is a random walk whose summands are in the domain of attraction of a stable law, and also sums of the form $\left.\sum_{i=1}^{n} f\left(b_{n}^{-1} S_{i-1}-x, b_{n}^{-1} S_{i}-x\right)\right)$ but only when the random walk has second moments: see the book [6] of Borodin and Ibragimov. These results are somewhat related to our results, and are in a sense much stronger, but only when the basic process $X$ is stable, whereas here we consider general Lévy processes.

The paper is organized as follows: in Section 2 we set the notation and state the results pertaining to the processes $V^{n}(f)$ and $V^{\prime n}(f)$. Our concern of being as complete as possible leads to a rather large number of results corresponding to various situations, which are all encountered for the processes $\Pi^{n}(r, a)$ already. Section 3 is devoted to stating the results for the (less important) processes $\bar{V}^{n}(f)$ and $\bar{V}^{\prime n}(f)$. The proofs are given in Sections 4-7.

\section{The processes $V^{n}(f)$ AND $V^{\prime n}(f)$}

\subsection{General setting and notation}

Our Lévy process $X$ is defined on the filtered probability space $\left(\Omega, \mathcal{F},\left(\mathcal{F}_{t}\right)_{t>0}, \mathbb{P}\right)$ and we assume that $\left(\mathcal{F}_{t}\right)$ is the right-continuous filtration generated by $X$ and that $\mathcal{F}=\vee \mathcal{F}_{t}$. It has characteristics $(b, c, F)$ : this is the unique triple $b \in \mathbb{R}$, and $c \geq 0$, and $F$ a measure on $\mathbb{R}$ which does not charge $\{0\}$ and integrates $x \mapsto x^{2} \wedge 1$, such that

$$
\mathbb{E}\left(\mathrm{e}^{i u X_{t}}\right)=\exp t\left(i u b-\frac{c u^{2}}{2}+\int F(\mathrm{~d} x)\left(\mathrm{e}^{i u x}-1-i u x 1_{\{|x| \leq 1\}}\right)\right) .
$$

We denote by $X^{c}$ the continuous martingale part (the "Gaussian part") of $X$, and also by $\mu$ the jump measure of $X$ and by $\nu$ the predictable compensator of $\mu$, that is $\nu(\mathrm{d} s, \mathrm{~d} x)=\mathrm{d} s F(\mathrm{~d} x)$. We use the symbol $\star$ to denote the stochastic integral with respect to the measures $\mu$ and $\mu-\nu$ (cf. [7] for more details on this notation and the decomposition below). Then

$$
X_{t}=b t+X_{t}^{c}+\left(x 1_{\{|x| \leq 1\}}\right) \star(\mu-\nu)_{t}+\left(x 1_{\{|x|>1\}}\right) \star \mu_{t} .
$$

Recall that $X^{c}=\sigma W$, where $\sigma=\sqrt{c}$ and $W$ is standard Brownian motion.

For any function $f$ for which the following makes sense, we write

$$
F(f)=\int f(x) F(\mathrm{~d} x), \quad H_{t}(f)=\mathbb{E}\left(f\left(X_{t}\right)\right), \quad \Gamma_{t}(f)=H_{t}\left(f^{2}\right)-H_{t}(f)^{2} .
$$

The following functions $\phi_{r}$ will often occur:

$$
\phi_{r}(x)= \begin{cases}1 \bigwedge|x|^{r} & \text { if } 0<r<\infty \\ 1 & \text { if } r=0,\end{cases}
$$

and the set

$$
I=\left\{r \geq 0: F\left(\phi_{r}\right)<\infty\right\}
$$


plays a fundamental role. It is an interval of the form $[\alpha, \infty)$ or $(\alpha, \infty)$, for some $\alpha \in[0,2]$. We have $2 \in I$ always, and the process $X-X^{c}$ is locally of finite variation if and only if $1 \in I$, and $X$ has locally finitely many jumps if and only if $0 \in I$. Set

$$
\left.\begin{array}{l}
\bar{b}=b-\int_{\{|x| \leq 1\}} x F(\mathrm{~d} x), \quad X_{t}^{\prime}=\sum_{s \leq t} \Delta X_{s} \quad \text { if } 1 \in I \\
X^{\prime \prime}=X-X^{c} .
\end{array}\right\}
$$

So if $1 \in I$ we have $X_{t}^{\prime \prime}=\bar{b} t+X_{t}^{\prime}$, and $\bar{b}$ is the "genuine" drift.

Let us introduce several classes of functions on $\mathbb{R}$. First, for $r \in(0, \infty)$ we denote by $\mathcal{E}_{r}, \mathcal{E}_{r}^{\prime}, \mathcal{E}_{r}^{\prime \prime}$ and $\mathcal{E}_{r}^{\prime \prime \prime}$ the following sets of Borel functions;

$$
\left.\begin{array}{ll}
\mathcal{E}_{r}: \text { all } f \text { with polynomial growth and } f(x)=|x|^{r} \text { on a neighborhood of } 0 \\
\mathcal{E}_{r}^{\prime}: \text { all } f \text { with polynomial growth and } f(x) \sim|x|^{r} \text { as } x \rightarrow 0 \\
\mathcal{E}_{r}^{\prime \prime}: \text { all } f \text { locally bounded and with } f(x)=\mathrm{O}\left(|x|^{r}\right) \text { as } x \rightarrow 0 \\
\mathcal{E}_{r}^{\prime \prime \prime}: \text { all } f \text { locally bounded and with } f(x)=\mathrm{o}\left(|x|^{r}\right) \text { as } x \rightarrow 0 .
\end{array}\right\}
$$

We write $\mathcal{E}_{r}^{b}, \mathcal{E}_{r}^{\prime b}, \mathcal{E}_{r}^{\prime \prime b}$ and $\mathcal{E}_{r}^{\prime \prime \prime b}$ for the sets of bounded functions belonging to $\mathcal{E}_{r}, \mathcal{E}_{r}^{\prime}, \mathcal{E}_{r}^{\prime \prime}$ and $\mathcal{E}_{r}^{\prime \prime \prime}$ respectively. Finally, as usual $C^{p}$ denotes the set of $p$ times continuously differentiable functions (for $p \geq 0$ ), and $C^{0, F}$ is the set of functions that are $F$-a.e. continuous. We have $\mathcal{E}_{r} \subset \mathcal{E}_{r}^{\prime} \subset \mathcal{E}_{r}^{\prime \prime} \subset \mathcal{E}_{s}^{\prime \prime} \subset \mathcal{E}_{s}^{\prime \prime \prime}$ for $s<r$, and $\phi_{r} \in \mathcal{E}_{r}^{b} \cap C^{0}$.

If $f \in \mathcal{E}_{r}^{\prime \prime}$ and $r \in I$ we have $(|f| \wedge 1) * \nu_{t}<\infty$ for all $t$, hence $f * \mu_{t}<\infty$ a.s. for all $t$ as well. When $r \notin I$ and $f \in \mathcal{E}_{r}^{\prime}$, then $f * \mu_{t}=\infty$ a.s. for all $t>0$ (to see this, observe that if further $f \geq 0$, the Laplace transform of $f \star \mu_{t}$ is $\lambda \mapsto \exp -t \int_{\{|x| \leq 1\}}\left(1-\mathrm{e}^{-\lambda f(x)}\right) F(\mathrm{~d} x)=0$ for all $\left.\lambda\right)$. In connection with this one can also define the process

$$
\Sigma(f, \varphi)=(f \varphi) \star(\mu-\nu)+(f(1-\varphi)) \star \mu .
$$

Here, $\varphi$ denotes a function having $1_{[-a, a]} \leq \varphi \leq 1$ for some $a>0$ and having a compact support when $f$ is unbounded; the last integral above is always meaningful, and the first (stochastic) integral makes sense as soon as $f \in \mathcal{E}_{r}^{\prime \prime}$ for some $r$ such that $2 r \in I$.

Finally we give some miscellaneous notation. We denote by $U$ a generic $\mathcal{N}(0,1)$ variable, and by $m_{r}$ the $r$ th absolute moment of $U$. We say that a sequence of processes $Y^{n}$ "converges u.c.p." to a process $Y$, denoted $Y^{n} \stackrel{\text { u.c.p. }}{\longrightarrow} Y$ or $Y_{t}^{n} \stackrel{\text { u.c.p. }}{\longrightarrow} Y_{t}$, if $\sup _{s \leq t}\left|Y_{s}^{n}-Y_{s}\right| \stackrel{\mathbb{P}}{\longrightarrow} 0$ for all $t>0$.

Recall also (see e.g. [7]) that the stable convergence in law of $Y^{n}$ to $Y$, when each $Y^{n}$ is a càdlàg process on $(\Omega, \mathcal{F}, \mathbb{P})$ and $\mathcal{F}$ is the $\sigma$-field generated by all $X_{t}$ 's and $Y$ is a càdlàg process defined on an extension of the space $(\Omega, \mathcal{F}, \mathbb{P})$, means that $\mathbb{E}\left(Z g\left(Y^{n}\right)\right) \rightarrow \mathbb{E}(Z g(Y))$ for all bounded $\mathcal{F}$-measurable variable $Z$ and all bounded continuous function $g$ on the space of all càdlàg functions, endowed with the Skorokhod topology.

\subsection{Results: the laws of large numbers}

Theorem 2.1. (i) The processes $V^{n}(f)$ converge in probability to a limit $V(f)$, for the Skorokhod topology, in the following cases:

(a) with $V(f)=f \star \mu$, when

$$
\begin{aligned}
& {[a-1] f \in \mathcal{E}_{2}^{\prime \prime \prime} \cap C^{0, F}} \\
& {[a-2] f \in \mathcal{E}_{r}^{\prime \prime} \cap C^{0, F} \text { if } r \in I \cap(1,2] \text { and } c=0 ;} \\
& {[a-3] f \in \mathcal{E}_{11}^{\prime \prime \prime} \cap C^{0, F} \text { if } 1 \in I \text { and } c=0 ;} \\
& {[a-4] f \in \mathcal{E}_{r}^{\prime \prime} \cap C^{0, F} \text { if } r \in I \cap(0,1] \text { and } c=\bar{b}=0 .}
\end{aligned}
$$

(b) with $V(f)_{t}=f \star \mu_{t}+c t$, when $f \in \mathcal{E}_{2}^{\prime} \cap C^{0, F}$.

(c) with $V(f)_{t}=f \star \mu_{t}+|\bar{b}| t$, when $f \in \mathcal{E}_{1}^{\prime} \cap C^{0, F}$ and $c=0$ and $1 \in I$.

(ii) In particular this holds for $f \in \mathcal{E}_{r}^{\prime} \cap C^{0, F}$ when $r \in[2, \infty)$, or when $r \in I \cap[1,2)$ if $c=0$, or when $r \in I \cap(0,1)$ if $c=0$ and $\bar{b}=0$. 
(iii) If $r \notin I$, or if $r \in(0,2)$ and $c>0$, or if $r \in(0,1)$ and $\bar{b} \neq 0$, then $\frac{1}{\Delta_{n}} H_{\Delta_{n}}\left(\phi_{r}\right) \rightarrow \infty$ and, for any $f \in \mathcal{E}_{r}^{\prime}$, the processes $\frac{\Delta_{n}}{H_{\Delta_{n}}\left(\phi_{r}\right)} V^{n}(f)_{t}$ converge u.c.p. to $t$; in particular, $V^{n}(f)_{t} \stackrel{\mathbb{P}}{\longrightarrow}+\infty$ for all $t>0$.

When $f(x)=|x|^{r}$ the case (b) $(r=2)$ is of course well known (this is the convergence of the realized quadratic variation), and (a) for $r>2$ may be found in [11] for general semimartingales, and (c) $(r=1)$ is also well known because then $V(f)$ is the variation process of $X$.

(iii) is not really satisfactory, since the rate $\frac{1}{\Delta_{n}} H_{\Delta_{n}}\left(\phi_{r}\right)$ is not "explicit". Note that any $r>0$ satisfies either the conditions (ii), or the conditions (iii). In the most interesting case $c>0$ it is however possible to give a more explicit result. In this case we can also provide an LLN for $V^{\prime n}(f)$ :

Theorem 2.2. Assume that $c>0$.

(i) If $f \in \mathcal{E}_{r}^{\prime}$ for some $r \in(0,2)$ then $\Delta_{n}^{1-r / 2} V^{n}(f)_{t}$ converges u.c.p. to $c^{r / 2} m_{r}$. The same holds for $r \in[2, \infty)$ if there is no jump (i.e. $F=0$ ).

(ii) If $f$ is bounded then $\Delta_{n} V^{\prime n}(f)_{t}$ converges u.c.p. to $t \mathbb{E}(f(\sigma U))$.

Note that, apart from the condition $r<2$ in (i) when $F \neq 0$, these results do not depend on the jumps at all, and moreover in (i) the limit does not even depend on the function $f$ (subject to being in $\mathcal{E}_{r}^{\prime}$ ). Clearly (i) fails if we assume only $f \in \mathcal{E}_{r}^{\prime \prime}$.

We also have another LLN for $V^{n}(f)$ without normalization in some of the cases covered by (iii) of Theorem 2.1, provided we center $V^{n}(f)$ appropriately:

Theorem 2.3. Let either $1<r<2$, or $r \leq 1$ and $2 r \in I$ and $c=0$. If $f \in \mathcal{E}_{r}^{b} \cap C^{0, F}$ and $\varphi \equiv 1$, or if $f \in \mathcal{E}_{r} \cap C^{0, F}$ and $\varphi$ is continuous with $1_{[-a, a]} \leq \varphi \leq 1_{\left[-a^{\prime}, a^{\prime}\right]}$ for some $0<a<a^{\prime}<\infty$, the processes $V^{n}(f)_{t}-\frac{1}{\Delta_{n}} H_{\Delta_{n}}(f \varphi) t$ converge in probability, for the Skorokhod topology, to $\Sigma(f, \varphi)$.

Remark 1. This theorem overlaps with (i) of Theorem 2.1, but in the overlapping cases $\frac{1}{\Delta_{n}} H_{\Delta_{n}}(f \varphi)$ converges to $F(f \varphi)+|\bar{b}|$ when $r=1$ (and $c=0$ ), and to $F(f \varphi)$ otherwise, so the two results are consistent.

Remark 2. When $c>0$ and $1<r<2$ and $f \in \mathcal{E}_{r}^{\prime} \cap C^{0, F}$, we can apply the two previous theorems at once: one can rewrite (i) of Theorem 2.2 as $\Delta_{n}^{1-r / 2}\left(V^{n}(f)_{t}-\frac{t}{\Delta_{n}} H_{\Delta_{n}}(f)\right) \stackrel{\text { u.c.p. }}{\longrightarrow} 0$ (see Lem. 5.2 below), so one can view Theorem 2.3 in this case as the CLT associated with the LLN of Theorem 2.2, or perhaps rather as a "second order" LLN because the convergence takes place in probability.

Remark 3. One could also prove that the result holds for $f$ in $\mathcal{E}_{r}^{\prime \prime}$ instead of $\mathcal{E}_{r}^{\prime}$. This is in contrast with Theorem 2.4-(ii) below, which is analogous to the above in a sense, but for which we cannot substitute $\mathcal{E}_{1}^{\prime}$ with $\mathcal{E}_{1}^{\prime \prime}$.

\subsection{Results: the central limit theorem}

We now turn to the CLT, for which various versions occur. First there are CLTs associated with LLNs having a deterministic limit (possibly after centering): for $V^{\prime n}(f)$ that concerns Theorem 2.2-(ii), and for $V^{n}(f)$ for $f \in \mathcal{E}_{r}^{\prime}$ that concerns the cases of (iii) of Theorem 2.1 not covered by Theorem 2.3, that is

(1) $c=0, r<1$ and $2 r \notin I$;

(2) $c>0, r=1$

(3) $c>0, r<1$.

For (1) the answer is complete, but probably useless because the rate is not explicit. For (2) the answer is half-way between a genuine CLT and an LNN: these two cases are dealt with in Theorem 2.4. Case (3) is considered later, together with the CLT about $V^{\prime n}(f)$.

Second, there are CLTs associated with LLN having random limits, that is (i) of Theorem 2.1. When $c>0$ we have such a CLT below, which extends results of [8]; this concerns the cases $r=2$ and $r>3$ only. When $c=0$ if a CLT exists it is very complicated, and indeed it does not exist in general (see [9] for special examples of this situation): we do not consider those cases here at all. 


\section{Theorem 2.4.}

(i) Let $f \in \mathcal{E}_{r}$ for some $r \in(0,1)$ and $c=0$ and $2 r \notin I$. Then $\frac{1}{\Delta_{n}} \Gamma_{\Delta_{n}}\left(\phi_{r}\right) \rightarrow \infty$, and the processes $\sqrt{\frac{\Delta_{n}}{\Gamma_{\Delta_{n}}\left(\phi_{r}\right)}}\left(V^{n}(f)_{t}-\frac{t}{\Delta_{n}} H_{\Delta_{n}}\left(\phi_{r}\right)\right)$ converge stably in law to a standard Brownian motion independent of $X$.

(ii) If $c>0$ and either $f \in \mathcal{E}_{1}^{b} \cap C^{0, F}$ and $\varphi=1$, or $f \in \mathcal{E}_{1} \cap C^{0, F}$ and $\varphi$ is continuous with $1_{[-a, a]} \leq$ $\varphi \leq 1_{\left[-a^{\prime}, a^{\prime}\right]}$ for some $0<a<a^{\prime}<\infty$, the processes $V^{n}(f)_{t}-\frac{t}{\Delta_{n}} H_{\Delta_{n}}(f \varphi)$ converge stably in law to the process $\Sigma(f, \varphi)+W^{\prime}$, where $W^{\prime}$ is a Wiener process independent of $X$ and with unit variance $c\left(m_{2}-m_{1}^{2}\right)=c(1-2 / \pi)$.

Remark 4. In the situation of (i) above, $\frac{\Delta_{n}}{H_{\Delta_{n}}\left(\phi_{r}\right)}\left(V^{n}(f)_{t}-\frac{t}{\Delta_{n}} H_{\Delta_{n}}\left(\phi_{r}\right)\right) \stackrel{\text { u.c.p. }}{\longrightarrow} 0$ by Theorem 2.1-(ii), hence we also have $H_{\Delta_{n}}\left(\phi_{r}\right) / \sqrt{\Gamma_{\Delta_{n}}\left(\phi_{r}\right)} \rightarrow \infty$.

Next we give the CLT associated with Theorem 2.2, with $r<1$ in (i) (case (3) above). Observe the two different centerings below.

Theorem 2.5. Assume that $c>0$.

(i) Let $f \in \mathcal{E}_{r}$ for some $r$ in $(0,1)$ in general, and some $r \in(0, \infty)$ if there is no jump.

1) The processes $\frac{1}{\sqrt{\Delta_{n}}}\left(\Delta_{n}^{1-r / 2} V^{n}(f)_{t}-t \Delta_{n}^{-r / 2} H_{\Delta_{n}}\left(\phi_{r}\right)\right)$ converge stably in law to a Wiener process independent of $X$ and with unit variance $c^{r}\left(m_{2 r}-m_{r}^{2}\right)$, and if further $f$ is bounded we can substitute $H_{\Delta_{n}}\left(\phi_{r}\right)$ with $H_{\Delta_{n}}(f)$.

2) If $1 \in I$ the same result holds for the processes $\frac{1}{\sqrt{\Delta_{n}}}\left(\Delta_{n}^{1-r / 2} V^{n}(f)_{t}-t c^{r / 2} m_{r}\right)$, whereas $\frac{1}{\Delta_{n}^{1-s / 2}}\left(\Delta_{n}^{1-r / 2} V^{n}(f)_{t}-\right.$ $\left.t c^{r / 2} m_{r}\right) \stackrel{\text { u.c.p. }}{\longrightarrow} 0$ if $1 \notin I$ and $s \in I$ for some $s \in(1,2)$.

(ii) Let $f$ be a bounded function.

1) The processes $\frac{1}{\sqrt{\Delta_{n}}}\left(\Delta_{n} V^{\prime n}(f)_{t}-t \mathbb{E}\left(f\left(X_{\Delta_{n}} / \sqrt{\Delta_{n}}\right)\right)\right)$ converge stably in law to a Wiener process independent of $X$ and with unit variance the variance of $f(\sigma U)$.

2) Assume further that $f$ is an even function. If $1 \in I$ the same result holds for the processes $\frac{1}{\sqrt{\Delta_{n}}}\left(\Delta_{n} V^{\prime n}(f)_{t}\right.$ $-t \mathbb{E}(f(\sigma U)))$, whereas $\frac{1}{\Delta_{n}^{1-s / 2}}\left(\Delta_{n} V^{\prime n}(f)_{t}-t \mathbb{E}(f(\sigma U))\right) \stackrel{\text { u.c.p. }}{\longrightarrow} 0$ if $1 \notin I$ and $s \in I$ for some $s \in(1,2)$.

Apart from the restriction $r<1$ in (i), we thus see that the presence of jumps does not affect the CLT. But it is important to note that the centering is not the one expected from Theorem 2.2 if there are jumps, unless $1 \in I$. The last claims in (2) of both (i) and (ii) are clearly not sharp, but we do not know the exact rate of convergence in those cases.

Finally we give a CLT associated with LLNs having random limits, when $c>0$. For this we need some more notation. We consider a sequence $\left(U_{n}\right)_{n \geq 1}$ of i.i.d. $\mathcal{N}(0,1)$ variables and also a standard Brownian motion $W^{\prime}$ independent of that sequence, all of these defined on an extension of $\left(\Omega, \mathcal{F},\left(\mathcal{F}_{t}\right)_{t \geq 0}, \mathbb{P}\right)$ and independent of $X$. Denote also an enumeration of the jump times of $X$ by $\left(T_{n}\right)_{n \geq 1}$, where the $\bar{T}_{n}$ 's are stopping times (not necessarily increasing in $n$, of course).

Theorem 2.6.

a) Assume that $c>0$ and let $g \in \mathcal{E}_{1}^{\prime \prime}$. Then the process (recall $\sigma=\sqrt{c}$ )

$$
Z(g)_{t}=\sum_{p: T_{p} \leq t} \sigma g\left(\Delta X_{T_{p}}\right) U_{p}
$$

is well defined, and when $F\left(g^{2}\right)<\infty$ it is a locally square-integrable martingale (on the extended space) w.r.t. the filtration generated by the triple $\left(X, Z, W^{\prime}\right)$.

b) If $f$ is $C^{1}$ over $\mathbb{R}$ and $C^{2}$ on a neighborhood of 0 , with $f^{\prime \prime}(x)=o(|x|)$ as $x \rightarrow 0$ ( $f^{\prime}$ and $f$ " denote the first and second derivatives), the processes $\frac{1}{\sqrt{\Delta_{n}}}\left(V^{n}(f)-V(f)^{(n)}\right)$ converge stably in law toward $Z\left(f^{\prime}\right)$.

c) If $f \in \mathcal{E}_{2}$ is $C^{1}$, the processes $\frac{1}{\sqrt{\Delta_{n}}}\left(V^{n}(f)_{t}-V(f)_{\Delta_{n}\left[t / \Delta_{n}\right]}\right)$ converge stably in law toward $Z\left(f^{\prime}\right)+c \sqrt{2} W^{\prime}$. 
Remark 5. We do not have convergence in law of $\frac{1}{\sqrt{\Delta_{n}}}\left(V^{n}(f)-V(f)\right)$, because of a peculiarity of Skorokhod topology: indeed if $X$ has a jump at some time $S$, then the above process has a "big" jump at $S$, and another "big" one at $\Delta_{n}\left[S / \Delta_{n}\right]$, and those two times are close but distinct. But if we substitute $V(f)_{t}$ with $V(f)_{\Delta_{n}\left[t / \Delta_{n}\right]}$ then those two jumps are put together at the single time $\Delta_{n}\left[S / \Delta_{n}\right]$, thus Skorokhod convergence may, and indeed does, occur. However the processes $\frac{1}{\sqrt{\Delta_{n}}}\left(V^{n}(f)-V(f)\right)$ converge finite-dimensionally, stably in law, to the limit given above.

Remark 6. In (b) the assumption is a bit more than asking $f \in \mathcal{E}_{3}^{\prime \prime \prime} \cap C^{1}$. This theorem says nothing when $f \in \mathcal{E}_{r} \cap C^{1}$ for some $2<r \leq 3$ and in these cases the sequence $\frac{1}{\sqrt{\Delta_{n}}}\left(V^{n}(f)-V(f)^{(n)}\right)$ is tight if $r=3$, but not tight if $2<r<3$. To see this, suppose that there is no jump and take $f \in \mathcal{E}_{r} \cap C^{0, F}$; then the variables $\frac{1}{\sqrt{\Delta_{n}}}\left(V^{n}(f)_{t}-V(f)_{t}^{(n)}\right)=\frac{1}{\sqrt{\Delta_{n}}} V^{n}(f)_{t}$ converge in probability to $+\infty$ when $2<r<3$, and to $c^{3 / 2} m_{3} t$ when $r=3$, and to 0 when $r>3$, by Theorem 2.2. When $r=2$, though, we have $V(f)_{t}=c t$ and then the previous theorem reduces to Theorem 2.5-(a), when $F=0$ and $r=2$.

One can also point out that when $f \in \mathcal{E}_{2}^{\prime} \backslash \mathcal{E}_{2}$ is $C^{1}$ we do not know whether the claim (c) holds true.

Finally, for practical purposes we need a multidimensional CLT in which we consider components as in (i) and (ii) of Theorem 2.5 and also as in Theorem 2.6. Below we have a $d$-dimensional process and the index set $\{1, \ldots, d\}$ for the components is partitioned into three (possibly empty) subsets $J, J^{\prime}$ and $J^{\prime \prime}$.

Theorem 2.7. Assume that $c>0$. Let $Y^{n}=\left(Y^{n, j}\right)_{1 \leq j \leq d}$ be the process having the following components:

- If $j \in J$, then $Y^{n, j}=\frac{1}{\sqrt{\Delta_{n}}}\left(V^{n}\left(f_{j}\right)-V\left(f_{j}\right)^{(n)}\right)$, where $f_{j}$ is like in Theorem 2.6-(b).

- If $j \in J^{\prime}$, then $Y_{t}^{n, j}=\frac{1}{\sqrt{\Delta_{n}}}\left(\Delta_{n}^{1-r(j) / 2} V^{n}\left(f_{j}\right)_{t}-t \Delta_{n}^{-r(j) / 2} H_{\Delta_{n}}\left(\phi_{r(j)}\right)\right)$, where $f_{j} \in \mathcal{E}_{r(j)}$ for some $r(j) \in(0,1)$ in general, or $r(j) \in(0, \infty)$ if there is no jump.

- If $j \in J^{\prime \prime}$, then $Y_{t}^{n, j}=\frac{1}{\sqrt{\Delta_{n}}}\left(\Delta_{n} V^{\prime n}\left(f_{j}\right)_{t}-t \mathbb{E}\left(f_{j}\left(X_{\Delta_{n}} / \sqrt{\Delta_{n}}\right)\right)\right)$, where $f_{j}$ is bounded.

Then $Y^{n}$ converges stably in law to a process $Y$ whose components $Y^{j}$ for $j \in J$ are of the form $Z\left(f_{j}^{\prime}\right)$ given by (2.9) with the same family $\left(U_{p}\right)$ for all $j$, and the components of $Y$ in $J^{\prime} \cup J^{\prime \prime}$ form a Wiener process independent of $X$ and of the $\left(U_{p}\right)$ 's and with unit variance-covariance given by

$$
c^{\prime j k}= \begin{cases}c^{r(j) / 2+r(k) / 2}\left(m_{r(j)+r(k)}-m_{r(j)} m_{r(k)}\right), & j, k \in J^{\prime} \\ \mathbb{E}\left(f_{j}(\sigma U) f_{k}(\sigma U)\right)-\mathbb{E}\left(f_{j}(\sigma U)\right) \mathbb{E}\left(f_{k}(\sigma U)\right) & j, k \in J^{\prime \prime} \\ \mathbb{E}\left(|\sigma U|^{r(j)} f_{k}(\sigma U)\right)-c^{r(j) / 2} \mathbb{E}\left(|U|^{r(j)} f_{k}(\sigma U)\right) & j \in J^{\prime}, k \in J^{\prime \prime} .\end{cases}
$$

Moreover we can replace $\Delta_{n}^{-r(j) / 2} H_{\Delta_{n}}\left(\phi_{r(j)}\right)$ by $c^{r / 2} m_{r}$ for $j \in J^{\prime}$ and $\mathbb{E}\left(f_{j}\left(X_{\Delta_{n}} / \sqrt{\Delta_{n}}\right)\right)$ by $\mathbb{E}\left(f_{j}(\sigma U)\right)$ for $j \in J^{\prime \prime}$, as soon as $1 \in I$ and provided also that $f_{j}$ is an even function for all $j \in J^{\prime \prime}$.

\section{The processes $\bar{V}^{n}(f)$ AND $\bar{V}^{\prime n}(f)$}

Now we give the results for the processes $\bar{V}^{n}(f)$ and $\bar{V}^{\prime n}(f)$ introduced in (1.5) and (1.6). As said in the introduction, this is of interest only when $T_{n}=n \Delta_{n}$ goes to $\infty$, an assumption made throughout.

The situation is much simpler than in the previous section, due to the built-in ergodic properties of $X$, or rather of its increments, and the proofs essentially boil down to using the usual LLN and CLT: we never encounter random limits in the LLNs. Moreover since $T_{n} \rightarrow \infty$ the stable convergence in law is automatically implied by the mere convergence in law, so we do not even state it in the results. The price we have to pay is that the variable $f\left(X_{\Delta_{n}}\right)$ should be integrable, or square-integrable. To avoid complicated statements, we just suppose that $f$ is bounded. Also, because of this necessary ergodicity, the results certainly cannot be extended to general semimartingales, contrarily to what happens for the results of the previous section.

We begin with the LLN, which corresponds to Theorems 2.1 and 2.2. 
Theorem 3.1. Assume $T_{n} \rightarrow \infty$.

(i) The processes $\frac{1}{T_{n}} \bar{V}^{n}(f)$ converge u.c.p. to a limit $v(f) t$ in the following cases:

(a) with $v(f)=F(f)$, when $f$ is bounded and like in [a-1]-[a-4] of Theorem 2.1;

(b) with $v(f)=F(f)+c$, when $f \in \mathcal{E}_{2}^{\prime b} \cap C^{0, F}$;

(c) with $v(f)=F(f)+|\bar{b}|$, when $f \in \mathcal{E}_{1}^{\prime b} \cap C^{0, F}$ and $c=0$ and $1 \in I$.

(ii) If $c>0$ and $f \in \mathcal{E}_{r}^{\prime b}$ for some $r \in(0,2)$, the processes $\frac{1}{n \Delta_{n}^{r / 2}} \bar{V}^{n}(f)_{t}$ converge u.c.p. to $c^{r / 2} m_{r} t$. The same holds when $r \in[2, \infty)$ if there is no jump.

(iii) If $c>0$ and $f$ is bounded the processes $\frac{1}{n} \bar{V}^{\prime n}(f)$ converge u.c.p. to $t \mathbb{E}(f(\sigma U))$.

As for the CLT, we have first a "general" result which covers all situations but is hardly usable in practice because the rate in some cases and the centering in most cases are not explicit:

Theorem 3.2. Assume $T_{n} \rightarrow \infty$.

(i) If either $f \in \mathcal{E}_{1}^{\prime \prime \prime b} \cap C^{0, F}$, or $f \in \mathcal{E}_{r} \cap C^{0, r}$ for some $r<1$ with $2 r \in I$ and $c=0$, the processes $\frac{1}{\sqrt{T_{n}}}\left(\bar{V}^{n}(f)_{t}-n H_{\Delta_{n}}(f) t\right)$ converge in law to a Wiener process with unit variance $F\left(f^{2}\right)$.

(ii) If $f \in \mathcal{E}_{1}^{\prime b} \cap C^{0, F}$ the processes $\frac{1}{\sqrt{T_{n}}}\left(\bar{V}^{n}(f)_{t}-n H_{\Delta_{n}}(f) t\right)$ converge in law to a Wiener process with unit variance $F\left(f^{2}\right)+c\left(m_{2}-m_{1}^{2}\right)=F\left(f^{2}\right)+c(1-2 / \pi)$.

(iii) If $r<1$ and either $c>0$ or $c=0$ and $2 r \notin I$, the processes $\frac{1}{\sqrt{n \Gamma_{\Delta_{n}}(f)}}\left(\bar{V}^{n}(f)_{t}-n H_{\Delta_{n}}(f) t\right)$ converge in law to a standard Brownian motion, for all $f \in \mathcal{E}_{r}^{b}$.

Finally we have a CLT similar to Theorem 2.5, and for which we give the multidimensional version only. Below the index set $\{1, \cdots, d\}$ is partitioned into two subsets $J^{\prime}$ and $J^{\prime \prime}$. Notice the difference with Theorem 2.3: the first set $J$ of components is absent here, although probably a result similar to Theorem 2.6 hold here (but with a limit which is a Wiener process again, instead of a discontinuous process).

Theorem 3.3. Assume $T_{n} \rightarrow \infty$ and $c>0$. Let $Y^{n}=\left(Y^{n, j}\right)_{1 \leq j \leq d}$ be the process having the following components:

- If $j \in J^{\prime}$, then $Y_{t}^{n, j}=\sqrt{n}\left(\frac{1}{n \Delta_{n}^{r(j) / 2}} \bar{V}^{n}\left(f_{j}\right)_{t}-t \Delta_{n}^{-r(j) / 2} H_{\Delta_{n}}\left(\phi_{r(j)}\right)\right)$, where $f_{j} \in \mathcal{E}_{r(j)}$ for some $r(j) \in$ $(0,1)$ in general or $r(j) \in(0, \infty)$ if there is no jump.

- If $j \in J^{\prime \prime}$, then $Y_{t}^{n, j}=\sqrt{n}\left(\frac{1}{n} \bar{V}^{\prime n}\left(f_{j}\right)_{t}-t \mathbb{E}\left(f_{j}\left(X_{\Delta_{n}} / \sqrt{\Delta_{n}}\right)\right)\right)$, where $f_{j}$ is bounded.

Then $Y^{n}$ converges stably in law to a Wiener process with unit variance-covariance given by (2.10).

Moreover we can replace $\Delta_{n}^{-r(j) / 2} H_{\Delta_{n}}\left(\phi_{r(j)}\right)$ by $c^{r / 2} m_{r}$ for a $j \in J^{\prime}$ it there is $s \in I \cap[0,1)$ such that the sequence $T_{n} \Delta_{n}^{(1-s)(1-r(j))}$ is bounded; in a similar way, we can replace $\mathbb{E}\left(f_{j}\left(X_{\Delta_{n}} / \sqrt{\Delta_{n}}\right)\right)$ by $\mathbb{E}\left(f_{j}(\sigma U)\right)$ for all $j \in J^{\prime \prime}$ it there is $s \in I \cap[0,1)$ such that the sequence $T_{n} \Delta_{n}^{(1-s)}$ is bounded.

\section{Some technical tools}

\subsection{Estimates for $X^{\prime \prime}$}

Let us begin with some additional notation. Below, $C$ denotes a constant which may change from line to line and depend on the characteristics $(b, c, F)$, and sometimes on the index $r$.

With any process $Y$ we associate its discretized version $Y^{(n)}$ by

$$
Y_{t}^{(n)}:=Y_{\Delta_{n}\left[t / \Delta_{n}\right]}=Y_{0}+\sum_{i=1}^{\left[t / \Delta_{n}\right]} \Delta_{i}^{n} Y
$$

As soon as $Y$ is càdlàg, by a well known result $Y^{(n)}(\omega) \stackrel{\mathrm{Sk}}{\longrightarrow} Y(\omega)$ (convergence for the Skorokhod topology). 
Recalling the notation $X^{\prime}$ and $X^{\prime \prime}$ of (2.6), we have:

Lemma 4.1. We have $X_{t}^{\prime \prime} / \sqrt{t} \stackrel{\mathbb{P}}{\longrightarrow} 0$ as $t \rightarrow 0$, and also $X_{t}^{\prime} / t \stackrel{\mathbb{P}}{\longrightarrow} 0$ if $1 \in I$, and

$$
\mathbb{E}\left(\phi_{2}\left(X_{t}^{\prime \prime} / \sqrt{t}\right)\right)= \begin{cases}\mathrm{o}\left(t^{1-s / 2}\right) & \text { if } s \in I \cap(0,2] \\ \mathrm{O}(t) & \text { if } 0 \in I .\end{cases}
$$

Proof. If $s \in I$ with $s \leq 2$ the law of $X_{1}^{\prime \prime}$ belongs to the class $\mathcal{G}_{s}$ defined in the paper [1]. Hence $X_{t}^{\prime \prime} / \sqrt{t} \stackrel{\mathbb{P}}{\longrightarrow} 0$, resp. $X_{t}^{\prime} / t \stackrel{\mathbb{P}}{\longrightarrow} 0$ if $1 \in I$, follows from Lemma $3.1-\left(\right.$ a) of that paper applied with $Y=X^{\prime \prime}$ and $\beta=2$, resp. $Y=X^{\prime}$ and $\beta=1$.

In the same lemma, the estimate (4.2) is proved when the process $X$ is symmetrical and $s>0$, but a close look at the proof shows that it also holds in general for $s>0$. Finally when $0 \in I$ then $X_{t}^{\prime \prime}=\bar{b} t+X_{t}^{\prime}$ and $X^{\prime}$ is compound Poisson, so (4.2) is obvious.

\subsection{Convergence of triangular arrays}

First, we gather in the following lemma some classical results (see e.g. [7]) which will be heavily used below. Let $\left(\chi_{i}^{n}=\left(\chi_{i}^{n j}\right)_{1 \leq j \leq d}\right)$ be a rowwise i.i.d. array of $\mathbb{R}^{d}$-valued random variables, and let $Z_{t}^{n}=\sum_{i=1}^{\left[t / \Delta_{n}\right]} \chi_{i}^{n}$ be their partial sums.

Let now $Z$ be a $d$-dimensional Lévy process with characteristics $\left(b^{\prime}, c^{\prime}, F^{\prime}\right)$ : we have $b^{\prime}=\left(b^{\prime j}\right) \in \mathbb{R}^{d}$, and $c^{\prime}=\left(c^{\prime i j}\right)$ is a nonnegative symmetric matrix, and $F^{\prime}$ is a positive measure on $\mathbb{R}^{d}$. We also consider an arbitrary $C_{b}^{1}$ truncation function $\psi$ on $\mathbb{R}$ : it satisfies $\psi(x)=x$ on a neighborhood of 0 and has compact support.

\section{Lemma 4.2.}

a) If for some $t>0$ the sequence of variables $Z_{t}^{n}$ converges in law to a limit, then the processes $Z^{n}$ converge in law, for the Skorokhod topology, to a Lévy process.

b) We have $Z^{n} \stackrel{\lll}{\longleftrightarrow}$ if and only if for some (and then all) $C_{b}^{1}$ truncation function $\psi$ we have

$$
\left.\begin{array}{l}
\frac{1}{\Delta_{n}} \mathbb{E}\left(\psi\left(\chi_{1}^{n j}\right)\right) \rightarrow b^{\prime j}+\int\left(\psi\left(x^{j}\right)-x^{j} 1_{\left\{\left|x^{j}\right| \leq 1\right\}}\right) F^{\prime}(\mathrm{d} x), \quad 1 \leq j \leq d \\
\frac{1}{\Delta_{n}} \mathbb{E}\left(\psi\left(\chi_{1}^{n i}\right) \psi\left(\chi_{1}^{n j}\right)\right) \rightarrow c^{\prime i j}+\int \psi\left(x^{i}\right) \psi\left(x^{j}\right) F^{\prime}(\mathrm{d} x), \quad 1 \leq i, j \leq d \\
\frac{1}{\Delta_{n}} \mathbb{E}\left(f\left(\chi_{1}^{n}\right)\right) \rightarrow \int f(x) F^{\prime}(\mathrm{d} x), \quad f \quad \text { bounded } F^{\prime} \text {-a.e. continuous null around } 0 .
\end{array}\right\}
$$

Next we turn to the so-called stable convergence in law, which has been defined in Section 2.1. We use this kind of convergence in the following setting: we are in the situation of Lemma $4.2-(\mathrm{b})$ with $Z=Z^{\prime}+W^{\prime}$, where $Z^{\prime}$ is a Lévy process on the space $\left(\Omega, \mathcal{F},\left(\mathcal{F}_{t}\right)_{t \geq 0}, \mathbb{P}\right)$, with characteristics $\left(b^{\prime}, 0, F^{\prime}\right)$, and $W^{\prime}$ is a $d-$ dimensional Wiener process with unit variance-covariance matrix $c^{\prime}$, defined on an extension of the original space and independent of $Z^{\prime}$ : so $Z$ is a Lévy process with characteristics $\left(b^{\prime}, c^{\prime}, F^{\prime}\right)$.

Lemma 4.3. Let $Z=Z^{\prime}+W^{\prime}$ be as above, and suppose that $\chi_{i}^{n}$ is $\mathcal{F}_{i \Delta_{n}}$-measurable and satisfies the first two properties in (4.3) with $k_{n}=1 / \Delta_{n}$. If

$$
\begin{gathered}
\frac{1}{\Delta_{n}} \mathbb{P}\left(\left\|\chi_{1}^{n}-Z_{\Delta_{n}}^{\prime}\right\|>\varepsilon\right) \rightarrow 0 \quad \forall \varepsilon>0 \\
\frac{1}{\Delta_{n}} \mathbb{E}\left(\psi\left(\chi_{1}^{n j}\right) X_{\Delta_{n}}^{c}\right) \rightarrow 0
\end{gathered}
$$

for all $j=1, \cdots, d$, the processes $Z^{n}$ converge stably in law to $Z$, as defined above.

If further $c^{\prime}=0$ (equivalently $W^{\prime}=0$ ) (4.4) alone implies $Z^{n} \stackrel{\mathbb{P}}{\longrightarrow} Z=Z^{\prime}$. 
Proof. Set $U^{n}=Z^{n}-Z^{\prime(n)}$, and suppose for a moment that the following two properties hold:

$\left.\begin{array}{l}\text { under (4.4) and (4.5), the processes } U^{n} \text { converge stably in law to } W^{\prime} \\ \text { under (4.4) and if } c^{\prime}=0 \text {, the processes } U^{n} \text { converge in law to } 0 .\end{array}\right\}$.

We have $Z^{\prime n} \stackrel{\text { Sk }}{\longrightarrow} Z^{\prime}$ pointwise. Then the second part of (4.6) gives our second claim. The first part of (4.6) yields that the sequence $U^{n}$ is C-tight (that is, tight with all limiting processes being continuous), so obviously for any continuous bounded function $g$ on the Skorokhod space we have $g\left(Z^{\prime n}+U^{n}\right)-g\left(Z^{\prime}+U^{n}\right) \stackrel{\mathbb{P}}{\longrightarrow} 0$. If further $V$ is bounded $\mathcal{F}$-measurable, then $\mathbb{E}\left(g\left(Z^{n}\right) V\right)-\mathbb{E}\left(g\left(Z^{\prime}+U^{n}\right) V\right) \rightarrow 0$. Applying once more (4.6) and the fact that the map $u \mapsto g\left(Z^{\prime}(\omega)+u\right)$ (where $u$ is a càdlàg function) is continuous for the Skorokhod topology at any function $u$ which is continuous in time, and remembering the definition of stable convergence, we see that in fact $\mathbb{E}\left(g\left(Z^{n}\right) V\right) \rightarrow \mathbb{E}\left(g\left(Z^{\prime}+W^{\prime}\right) V\right)$, which is our first claim.

So it remains to prove (4.6). For this, we observe that $U_{t}^{n}=\sum_{i=1}^{\left[t / \Delta_{n}\right]} \zeta_{i}^{n}$, where the $\zeta_{i}^{n}$ 's for $i=1,2, \ldots$ are i.i.d. and distributed as $\chi_{1}^{n}-Z_{\Delta_{n}}^{\prime}$. First, $Z^{\prime(n)} \stackrel{\mathrm{Sk}}{\longrightarrow} Z^{\prime}$ as said before, and $Z^{\prime}$ has characteristics $\left(b^{\prime}, 0, F^{\prime}\right)$, hence Lemma 4.2 implies that the variables $Z_{\Delta_{n}}^{\prime}$ satisfy (4.3) with the same $b^{\prime}$ and $F^{\prime}$, and with $c^{\prime}=0$. Second, (4.4) obviously implies that the last part of (4.3) is satisfied by the variables $\zeta_{1}^{n}$ with $F^{\prime}=0$. Then consider the three properties:

$$
\begin{aligned}
\frac{1}{\Delta_{n}} \mathbb{E}\left(\psi\left(\zeta_{1}^{n j}\right)\right) & \rightarrow 0, \\
\frac{1}{\Delta_{n}} \mathbb{E}\left(\psi\left(\zeta_{1}^{n i}\right) \psi\left(\zeta_{1}^{n j}\right)\right) & \rightarrow c^{i j}, \\
\frac{1}{\Delta_{n}} \mathbb{E}\left(\psi\left(\zeta_{1}^{n j}\right) \psi\left(X_{\Delta_{n}}^{c}\right)\right) & \rightarrow 0 .
\end{aligned}
$$

By Lemma 4.2 we have the second part of (4.6) if (4.4), (4.7) and (4.8) with $c^{\prime}=0$ holds, and by the criterion given in Theorem IX.7.19 of [7] if we additionally have (4.9) then the first part of (4.6) holds.

The properties of $\psi$ yield a continuous bounded function $g$ vanishing around 0 , such that for all $\varepsilon>0$ small enough we have $\left|\psi\left(x-x^{\prime}\right)-\psi(x)+\psi\left(x^{\prime}\right)\right| \leq C 1_{\left\{\left|x-x^{\prime}\right|>\varepsilon\right\}}+\varepsilon g(x)$. Then (4.4) and the third part of (4.3) for $Z_{\Delta_{n}}^{\prime}$ yield

$$
\underset{n}{\limsup } \frac{1}{\Delta_{n}}\left(\mathbb{E}\left(\psi\left(\zeta_{1}^{n j}\right)\right)-\mathbb{E}\left(\psi\left(\chi_{1}^{n j}\right)\right)+\mathbb{E}\left(\psi\left(Z_{\Delta_{n}}^{\prime j}\right)\right)\right) \leq \varepsilon \int F^{\prime}(\mathrm{d} x) g\left(x^{j}\right) .
$$

$\varepsilon$ is arbitrarily small, hence the above limsup actually vanishes and, since $\frac{1}{\Delta_{n}} \mathbb{E}\left(\psi\left(Z_{\Delta_{n}}^{\prime j}\right)\right)$ and $\frac{1}{\Delta_{n}} \mathbb{E}\left(\psi\left(\chi_{1}^{n j}\right)\right)$ have the same limit, we deduce (4.7).

In a similar way, if $\psi_{\gamma}(x)=\gamma \psi(x / \gamma)$, we have for all $\gamma>0$ a continuous bounded function $g_{\gamma}$ vanishing around 0 , such that for all $\varepsilon>0$ small enough we have

$$
\begin{aligned}
\left|\psi\left(x^{i}-x^{i}\right) \psi\left(x^{j}-x^{\prime j}\right)-\psi\left(x^{i}\right) \psi\left(x^{j}\right)+\psi\left(x^{i i}\right) \psi\left(x^{\prime j}\right)\right| \leq C 1_{\left\{\left\|x-x^{\prime}\right\|>\varepsilon\right\}}+\varepsilon g_{\gamma}(\|x\|) \\
+\left|\psi_{\gamma}\left(x^{\prime i}\right)\right|\left(\left|\psi_{\gamma}\left(x^{j}\right)\right|+\left|\psi_{\gamma}\left(x^{\prime j}\right)\right|\right)+\left|\psi_{\gamma}\left(x^{\prime j}\right)\right|\left(\left|\psi_{\gamma}\left(x^{i}\right)\right|+\left|\psi_{\gamma}\left(x^{\prime i}\right)\right|\right) .
\end{aligned}
$$

Recall that $\frac{1}{\Delta_{n}} \mathbb{E}\left(\psi_{\gamma}\left(Z_{\Delta_{n}}^{\prime j}\right)^{2}\right) \rightarrow \int \psi_{\gamma}\left(x^{j}\right)^{2} F^{\prime}(\mathrm{d} x)$, which in turn goes to 0 as $\gamma \rightarrow 0$, whereas $\frac{1}{\Delta_{n}} \mathbb{E}\left(\psi_{\gamma}\left(\chi_{1}^{n j}\right)^{2}\right) \rightarrow$ $c^{\prime j j}+\int \psi_{\gamma}\left(x^{j}\right)^{2} F^{\prime}(\mathrm{d} x)$, which stays bounded as $\gamma \rightarrow 0$. Then using Cauchy-Schwarz and the same argument as for proving (4.7), we get by taking the $\lim \sup$ in $n$, then letting $\varepsilon \rightarrow 0$, then letting $\gamma \rightarrow 0$ :

$$
\left.\frac{1}{\Delta_{n}}\left(\mathbb{E}\left(\psi\left(\zeta_{i}^{n j}\right)\right) \psi\left(\zeta_{1}^{n j}\right)\right)-\mathbb{E}\left(\psi\left(\chi_{1}^{n i}\right) \psi\left(\chi_{1}^{n j}\right)\right)+\mathbb{E}\left(\psi\left(Z_{\Delta_{n}}^{\prime i}\right) \psi\left(Z_{\Delta_{n}}^{\prime j}\right)\right)\right) \rightarrow 0 .
$$

Then (4.8) follows. 
Finally we turn to (4.9). We $\left|\psi\left(x-x^{\prime}\right) \psi(y)-\psi(x) y+\psi\left(x^{\prime}\right) y\right| \leq C 1_{\left\{\left|x-x^{\prime}\right|>\varepsilon\right\}}+\varepsilon g(x)+C|y| 1_{\{|y|>\gamma\}}$, for some $\gamma>0$. So just as before, with in addition the fact that $\mathbb{E}\left(\left|X_{\Delta_{n}}^{c}\right| 1_{\left\{\left|X_{\Delta_{n}}^{c}\right|>\gamma\right\}}\right) \leq C \Delta_{n}^{2}$ for any fixed $\gamma$, we get

$$
\frac{1}{\Delta_{n}} \mathbb{E}\left(\psi\left(\zeta_{1}^{n j}\right) \psi\left(X_{\Delta_{n}}^{c}\right)\right)-\frac{1}{\Delta_{n}} \mathbb{E}\left(\psi\left(\chi_{1}^{n j}\right) X_{\Delta_{n}}^{c}\right)+\frac{1}{\Delta_{n}} \mathbb{E}\left(\psi\left(Z_{\Delta_{n}}^{\prime j}\right) X_{\Delta_{n}}^{c}\right) \rightarrow 0
$$

Since the last term in the left side above vanishes (because $X^{c}$ is independent of $Z^{\prime}$ ), we deduce (4.9) from (4.5), and the proof is finished.

\section{TheOREM 2.1 AND SOME CONSEQUENCES}

\subsection{Proof of Theorem 2.1-(i, ii)}

Before proceeding to the proof, let us introduce a family $\left(\psi_{\eta}\right)_{\eta>0}$ of $C^{2}$ functions satisfying

$$
1_{[-\eta, \eta]}(|x|) \leq \psi_{\eta}(x) \leq 1_{[-2 \eta, 2 \eta]}(|x|), \quad\left|\psi_{\eta}^{\prime}\right| \leq C / \eta, \quad\left|\psi_{\eta}^{\prime \prime}\right| \leq C / \eta^{2}
$$

(take for example $\psi_{\eta}(x)=\psi(x / \eta)$, where $\psi$ is $C^{2}$ with $1_{[-1,1]}(|x|) \leq \psi(x) \leq 1_{[-2,2]}(|x|)$ and bounded derivatives). Next, the proof goes through a number of steps.

Step 1. (ii) is a particular case of (i), and if $f$ satisfies either one of the conditions in (i) the process $f \star \mu$ is well defined and càdlàg, hence $V(f)$ as well. In view of the convergence $V(f)^{(n)} \stackrel{\mathrm{Sk}}{\longrightarrow} V(f)$, which holds $\omega$-wise, it is enough to prove the following:

$$
\bar{Z}^{n}(f):=V^{n}(f)-V(f)^{(n)} \stackrel{\text { u.c.p. }}{\longrightarrow} 0 .
$$

Step 2. Here we prove (5.2) when $f \in C^{0, F}$ vanishes on a neighborhood of 0 , say $[-2 \varepsilon, 2 \varepsilon]$. We introduce a series of notation to be used later again. Let $S_{1}, S_{2}, \cdots$ be the successive jump times of $X$ with absolute size bigger than $\varepsilon$, and $R_{p}=\Delta X_{S_{p}}$ (these depend on $\varepsilon$, of course); we put

$$
X(\varepsilon)_{t}=X_{t}-\left(x 1_{\{|x|>\varepsilon\}}\right) * \mu_{t}=X_{t}-\sum_{p: S_{p} \leq t} R_{p}
$$

and $R_{p}^{\prime n}=\Delta_{i}^{n} X(\varepsilon)$ on the set $\left\{S_{p} \in\left((i-1) \Delta_{n}, i \Delta_{n}\right]\right.$. Finally if $T>0$ let $\Omega_{n}(T, \varepsilon)$ be the set of all $\omega$ such that each interval $[0, T] \cap\left((i-1) \Delta_{n}, i \Delta_{n}\right]$ contains at most one $S_{p}(\omega)$, and such that $\left|\Delta_{i}^{n} X(\varepsilon)(\omega)\right| \leq 2 \varepsilon$ for all $i \leq T / \Delta_{n}$. Then $\Omega_{n}(T, \varepsilon) \rightarrow \Omega$ as $n \rightarrow \infty$.

Recalling $f(x)=0$ when $|x| \leq 2 \varepsilon$, we see that on the set $\Omega_{n}(T, \varepsilon)$ and for all $t \leq T$,

$$
V^{n}(f)_{t}-V(f)_{t}^{(n)}=\sum_{p: S_{p} \leq \Delta_{n}\left[t / \Delta_{n}\right]}\left(f\left(R_{p}+R_{p}^{\prime n}\right)-f\left(R_{p}\right)\right)
$$

Since $f$ is $F$-a.e. continuous there is a null set $N$ such that, if $\omega \notin N$, then $f$ is continuous at each point $R_{p}(\omega)$, whereas $R_{p}^{\prime n}(\omega) \rightarrow 0$, so $\sup _{t \leq T}\left|V^{n}(f)_{t}-V(f)_{t}^{(n)}\right| \rightarrow 0$ when $\omega \notin N$. Hence (5.2) is obvious (we even have almost sure convergence).

Step 3. For each $r$ let $f_{r}$ be a given nonnegative function with compact support and $C^{2}$ outside any neighborhood of 0 and satisfying $f_{r}(x)=|x|^{r}$ on some neighborhood of 0 . Here we prove that it is enough to have the following property: (5.2) holds for $f_{r}$, when $r=2$, or $r \in I \cap[1,2)$ and $c=0$, or $r \in I \cap(0,1)$ and $c=\bar{b}=0$ : we assume this below. 
Let $f \in \mathcal{E}_{r}^{\prime \prime \prime} \cap C^{0, F}$ with $r$ as above. For any $\eta>0$ the function $f\left(1-\psi_{\eta}\right)$ satisfies (5.2) with $V\left(f\left(1-\psi_{\eta}\right)\right)=$ $\left(f\left(1-\psi_{\eta}\right)\right) * \mu$ by Step 2 , and we have $\left|f \psi_{\eta}\right| \leq \varepsilon_{\eta} f_{r}$ with $\varepsilon_{\eta}$ going to 0 as $\eta \rightarrow 0$. Then $\left|\bar{Z}^{n}\left(f \psi_{\eta}\right)\right| \leq$ $\varepsilon_{\eta}\left(\left|\bar{Z}^{n}\left(f_{r}\right)\right|+\left|V\left(f_{r}\right)^{(n)}\right|\right)$. Since $f_{r}$ satisfies (5.2) by hypothesis, and $V\left(f_{r}\right)^{(n)} \rightarrow V\left(f_{r}\right)$, by letting $\eta \rightarrow 0$ we readily deduce (5.2) with $V(f)=f * \mu$ for $f$. This proves [a-1] and [a-3] and, since $f-f_{r}$ belongs to $\mathcal{E}_{r}^{\prime \prime}$ as soon as $f \in \mathcal{E}_{r}$, this also proves (b) and (c).

Finally let $f \in \mathcal{E}_{r}^{\prime \prime} \cap C^{0, F}$ in cases [a-2] or [a-4]. On the one hand $f\left(1-\psi_{\eta}\right)$ and $f_{r}\left(1-\psi_{\eta}\right)$ satisfy (5.2) by Step 2, and $f_{r}$ does by hypothesis, hence $f_{r} \psi_{\eta}$ does as well. On the other hand $\left|f \psi_{\eta}\right| \leq C f_{r} \psi_{\eta}$ for some constant $C$, hence $\left|\bar{Z}^{n}\left(f \psi_{\eta}\right)\right| \leq\left|\bar{Z}^{n}\left(f_{r} \psi_{\eta}\right)\right|+V\left(f_{r} \psi_{\eta}\right)^{(n)}$. Since $V\left(f_{r} \psi_{\eta}\right)^{(n)}$ goes to 0 locally uniformly in time as $\eta \rightarrow 0$, we get (5.2) for $f$.

Step 4. We are left to prove (5.2) when $f=f_{r}$ as given in Step 3, with $r$ as above. Set

$$
g(x, y)=f(x+y)-f(x)-f(y)-x f^{\prime}(y) 1_{\{|x| \leq 1\}}, \quad k(x, y)=f(x+y)-f(x)-f(y)
$$

with the convention $f^{\prime}(0)=0$ if $r \leq 1$ (otherwise, $f^{\prime}(0)$ is the derivative of $f$ at 0 , of course). If we apply the usual Itô's formula if $r=2$ (so $f$ is $C_{b}^{2}$ over $\mathbb{R}$ ) and its extension given in Theorem 3.1 of [10] if $r<2$, we see that $f(X)-V(f)=A+M$, where $A_{t}=\int_{0}^{t} \alpha\left(X_{s}\right) d s$ and $M$ is a locally square-integrable martingale, all these given by

$$
\begin{gathered}
\alpha(y)= \begin{cases}b f^{\prime}(y)+\frac{c}{2}\left(f^{\prime \prime}(y)-f^{\prime \prime}(0)\right)+\int F(\mathrm{~d} x) g(x, y) & \text { if } r=2 \\
b f^{\prime}(y)+\int F(\mathrm{~d} x) g(x, y) & \text { if } 1<r<2, c=0 \\
\bar{b} f^{\prime}(y)-|\bar{b}|+\int F(\mathrm{~d} x) k(x, y) & \text { if } r=1, c=0 \\
\int F(\mathrm{~d} x) k(x, y) & \text { if } 0<r<1, c=\bar{b}=0,\end{cases} \\
M_{t}= \begin{cases}\int_{0}^{t} f^{\prime}\left(X_{s}\right) \mathrm{d} X_{s}^{c}+k\left(x, X_{-}\right) \star(\mu-\nu)_{t} & \text { if } r=2 \\
k\left(x, X_{-}\right) \star(\mu-\nu)_{t} & \text { if } r<2 .\end{cases}
\end{gathered}
$$

Moreover the predictable bracket of $M$, say $A^{\prime}$, has the form $A_{t}^{\prime}=\int_{0}^{t} \alpha^{\prime}\left(X_{s}\right) \mathrm{d} s$, where

$$
\alpha^{\prime}(y)= \begin{cases}c f^{\prime}(y)^{2}+\int F(\mathrm{~d} x) k(x, y)^{2} & \text { if } r=2 \\ \int F(\mathrm{~d} x) k(x, y)^{2} & \text { if } r<2 .\end{cases}
$$

Step 5. Observe that $Z^{n}(f)_{t}$ is the sum of $\left[t / \Delta_{n}\right]$ i.i.d. summands, distributed as $\zeta_{n}=f\left(X_{\Delta_{n}}\right)-V(f)_{\Delta_{n}}=$ $A_{\Delta_{n}}+M_{\Delta_{n}}$. Since $\mathbb{E}\left(M_{\Delta_{n}}\right)=0$, it is clear that (5.2) follows from the two properties

$$
\frac{1}{\Delta_{n}} \mathbb{E}\left(\left|A_{\Delta_{n}}\right|\right) \rightarrow 0, \quad \frac{1}{\Delta_{n}} \mathbb{E}\left(M_{\Delta_{n}}^{2}\right) \rightarrow 0 .
$$

But $\mathbb{E}\left(\left|A_{\Delta_{n}}\right|\right) \leq \int_{0}^{\Delta_{n}} \mathbb{E}\left(\left|\alpha\left(X_{t}\right)\right| \mathrm{d} t\right.$ and $\mathbb{E}\left(M_{\Delta_{n}}^{2}\right)=\int_{0}^{\Delta_{n}} \mathbb{E}\left(\alpha^{\prime}\left(X_{t}\right) \mid \mathrm{d} t\right.$. Then it is enough to prove that, as $t \rightarrow 0$

$$
|\alpha(y)| \leq C, \quad\left|\alpha^{\prime}(y)\right| \leq C, \quad \alpha\left(X_{t}\right) \stackrel{\mathbb{P}}{\longrightarrow} 0, \quad \alpha^{\prime}\left(X_{t}\right) \stackrel{\mathbb{P}}{\longrightarrow} 0 .
$$

Step 6. Finally we prove (5.7). Suppose first $r=2$, so $f$ is $C_{b}^{2}$ and $f(0)=f^{\prime}(0)=0$. Hence $|k(x, y)| \leq C \phi_{1}(x)$ and $|g(x, y)| \leq C \phi_{2}(x)$ and $\lim _{y \rightarrow 0} k(x, y)=\lim _{y \rightarrow 0} g(x, y)=0$. Then the first two properties of (5.7) are obvious, and by Lebesgue's theorem $\alpha(y) \rightarrow 0$ and $\alpha^{\prime}(0) \rightarrow 0$ as $y \rightarrow 0$, hence we get the last two properties as well.

Suppose next that $1<r<2$, so the function $f$ is $C_{b}^{1}$ and $f^{\prime}$ is Hölder with index $r-1$, and $f(0)=f^{\prime}(0)=0$, hence $|k(x, y)| \leq C \phi_{1}(x)$ and $|g(x, y)| \leq C \phi_{r}(x)$, and again $\lim _{y \rightarrow 0} k(x, y)=\lim _{y \rightarrow 0} g(x, y)=0$. Then we conclude (5.7) as above, upon using the assumption $r \in I$. 
Finally if $r \leq 1$ we have $|k(x, y)| \leq C \phi_{r}(x)$, and again $k(x, y) \rightarrow 0$ as $y \rightarrow 0$. Since $r \in I$ we conclude as above if $r<1$, or if $r=1$ and $\bar{b}=0$. If $r=1$ and $\bar{b} \neq 0$, in order to get $\alpha\left(X_{t}\right) \stackrel{\mathbb{P}}{\longrightarrow} 0$ we need an additional argument: we use Lemma 4.1 , which yields $X_{t}^{\prime} / t \stackrel{\mathbb{P}}{\longrightarrow} 0$, so if $\bar{b}>0$, say, then $\mathbb{P}\left(X_{t}>0\right) \rightarrow 1$ because $X_{t}=X_{t}^{\prime}+\bar{b} t$, and we have $f^{\prime}(y)=1$ if $y>0$, so the result follows. The same argument applies when $\bar{b}<0$, using $f^{\prime}(y)=-1$ if $y<0$.

\subsection{The behavior of $H_{t}(f)$}

We draw some consequences of the convergence $V^{n}(f) \stackrel{\mathbb{P}}{\longrightarrow} V(f)$ in Theorem 2.1-(i), which a fortiori implies $V^{n}(f) \stackrel{\lll}{\longleftrightarrow} V(f)$. Note that $V^{n}(f)_{t}$ is the sum of $\left[t / \Delta_{n}\right]$ i.i.d. variables distributed as $f\left(X_{\Delta_{n}}\right)$. Hence the first part of (4.3) applied with a truncation $\psi$ having $\psi(x)=x$ as soon as $|x| \leq \sup |f|$ when $f$ is bounded, plus the fact that the above convergence takes place for any sequence $\Delta_{n} \rightarrow 0$, yield the following, as $t \rightarrow 0$ and for any $f \in C^{0, F}:$

$$
\frac{1}{t} H_{t}(f) \rightarrow \begin{cases}c+F(f) & \text { if } f \in \mathcal{E}_{2}^{\prime b} \\
|\bar{b}|+F(f) & \text { if } f \in \mathcal{E}_{1}^{\prime \prime b} \\
F(f) & \text { if }\left\{\begin{array}{l}
f \in \mathcal{E}_{2}^{\prime \prime \prime} \\
f \in \mathcal{E}_{r}^{\prime \prime}, \quad 1<r<2, \quad r \in I, \quad c=0 \\
f \in \mathcal{E}_{1}^{\prime \prime \prime}, \quad 1 \in I, \quad c=0 \\
f \in \mathcal{E}_{r}^{\prime \prime}, \quad r<1, \quad r \in I, \quad c=\bar{b}=0 .
\end{array}\right.\end{cases}
$$

We deduce from these facts a number of useful properties.

Lemma 5.1. Assume $c=0$ and let $r \in(0,2)$. Then as $t \rightarrow 0$ :

$$
H_{t}\left(\phi_{r}\right)= \begin{cases}\mathrm{o}\left(t^{r / s}\right) & \text { if } r<s \text { with } s \in I \text { and further } \bar{b}=0 \text { when } s \leq 1 \\ \mathrm{O}\left(t^{r}\right) & \text { if } r \leq 1 \text { and } 1 \in I \text { and } \bar{b} \neq 0 \text { when } s \leq 1\end{cases}
$$

and for any $a>0$ and $s \in I \cap[0,2]$,

$$
\mathbb{E}\left(\phi_{r}\left(X_{t}\right) 1_{\left\{\left|X_{t}\right| \geq a \sqrt{t}\right\}}\right)= \begin{cases}\mathrm{o}\left(t^{1-s / 2+r / 2}\right) & \text { if } r<s \text { and either } s \geq 1 \\ & \text { or } s<1 \text { and } \bar{b}=0 \\ \mathrm{o}\left(t^{1-s / 2+r s / 2}\right) & \text { if } r<1,0<s \leq 1, \bar{b} \neq 0 \\ \mathrm{O}(t) & \text { in all other cases. }\end{cases}
$$

Proof. When $r \leq 1$, Hölder inequality yields $t^{-r} H_{t}\left(\phi_{r}\right) \leq\left(t^{-1} H_{t}\left(\phi_{1}\right)\right)^{r}$, hence the second part of (5.8) yields the second part of (5.9). Moreover if $\phi_{r, \varepsilon}(x)=|x|^{r} \wedge \varepsilon$, for $\varepsilon \in(0,1)$ and $r<s$ we have by Hölder inequality again

$$
t^{-r / s} H_{t}\left(\phi_{r}\right) \leq\left(t^{-1} H_{t}\left(\phi_{r, \varepsilon}^{s / r}\right)\right)^{r / s}+t^{-r / s} H_{t}\left(\phi_{r}-\phi_{r, \varepsilon}\right) .
$$

Since $\phi_{r}-\phi_{r, \varepsilon} \in \mathcal{E}_{2}^{b} \cap C^{0}$ the last term above goes to 0 for any $\varepsilon$ because $r<s$, by the third part of (5.8). Since $\phi_{r, \varepsilon}^{s / r} \in \mathcal{E}_{s}^{b} \cap C^{0}$, the first term on the right goes to $F\left(\phi_{r, \varepsilon}^{s / r}\right)$, which in turn goes to 0 as $\varepsilon \rightarrow 0$ because $s \in I$. Then we obtain the first part of (5.9).

Now if $r<s$ and either $s \geq 1$ or $s<1$ an $\bar{b}=0$, Hölder inequality yields

$$
\begin{aligned}
\mathbb{E}\left(\phi_{r}\left(X_{t}\right) 1_{\left\{X_{t} \geq a \sqrt{t}\right\}}\right) & \leq\left(\mathbb{E}\left(\phi_{s}\left(X_{t}\right)\right)\right)^{r / s}\left(\mathbb{P}\left(\left|X_{t}\right| \geq a \sqrt{t}\right)\right)^{1-r / s} \\
& \leq C_{a} H_{t}\left(\phi_{s}\right)^{r / s}\left(\mathbb{E}\left(\phi_{2}\left(X_{t} / \sqrt{t}\right)\right)\right)^{1-r / s}
\end{aligned}
$$


Then we apply (4.2) (we have $X=X^{\prime \prime}$ here), and also (5.8) which gives $H_{t}\left(\phi_{s}\right) \leq C t$, and we get the firts part of (5.10). When $s \leq 1$ and $\bar{b} \neq 0$ another application of Hölder inequality yields for $r \leq 1$ :

$$
\mathbb{E}\left(\phi_{r}\left(X_{t}\right) 1_{\left\{X_{t} \geq a \sqrt{t}\right\}}\right) \leq C_{a} H_{t}\left(\phi_{1}\right)^{r}\left(\mathbb{E}\left(\phi_{2}\left(X_{t} / \sqrt{t}\right)\right)\right)^{1-r}
$$

and we conclude the second part of (5.10), and also the third part when further $s=0$. Finally if $r \geq s$ and further $\bar{b}=0$ if $s \leq 1$ and $r<1$, the left side of (5.10) is smaller than $H_{t}\left(\phi_{r}\right)$, hence (5.8) gives the last part of (5.10) in these cases.

Lemma 5.2. Assume that $c>0$, and recall $m_{r}=\mathbb{E}\left(|U|^{r}\right)$, where $U$ is $\mathcal{N}(0,1)$. When, $t \rightarrow 0$ we have the following behaviors:

a) Let $f \in \mathcal{E}_{r}^{\prime b}$ for some $r \in(0,2)$ in general, or $f \in \mathcal{E}_{r}^{\prime}$ and $r>0$ if $X$ is continuous. Then

$$
t^{-r / 2} H_{t}(f) \rightarrow c^{r / 2} m_{r}, \quad t^{-1 / 2-r / 2} \mathbb{E}\left(X_{t}^{c} f\left(X_{t}\right)\right) \rightarrow 0 .
$$

b) Let $s \in I \cap[0,2]$ and let $f \in \mathcal{E}_{r}^{b}$ for some $r \in(0,1)$ in general, or $f \in \mathcal{E}_{r}$ and $r>0$ if $X$ is continuous. Then

$$
t^{-r / 2} H_{t}(f)-c^{r / 2} m_{r}= \begin{cases}\mathrm{O}(t) & \text { if } X \text { is continuous } \\ \mathrm{O}\left(t^{1-r / 2}\right) & \text { if } s=0 \text { and } r<1 \\ \mathrm{O}\left(t^{1-r / 2}\right) & \text { if } s \leq r<1 \text { and } \bar{b}=0 \\ \mathrm{O}\left(t^{1-s / 2}\right) & \text { if } r<1 \leq s, \text { or } \text { if } r<s<1 \text { and } \bar{b}=0 \\ \mathrm{o}\left(t^{1-s / 2-r / 2+r s / 2}\right) & \text { if } \bar{b} \neq 0,0<s \leq 1 .\end{cases}
$$

c) If $f$ is a bounded function we have

$$
\mathbb{E}\left(f\left(X_{t} / \sqrt{t}\right)\right) \rightarrow \mathbb{E}(f(\sigma U)), \quad t^{-1 / 2} \mathbb{E}\left(X_{t}^{c} f\left(X_{t} / \sqrt{t}\right)\right) \rightarrow 0 .
$$

If further $f$ is an even function, then

$$
\mathbb{E}\left(f\left(X_{t} / \sqrt{t}\right)\right)-\mathbb{E}(f(\sigma U))= \begin{cases}\mathrm{o}\left(t^{1-s / 2}\right) & \text { if } s \in I \cap(0,2) \\ \mathrm{O}(t) & \text { if } 0 \in I .\end{cases}
$$

d) With the assumptions of (a) and if $f^{\prime}$ is a bounded function we have

$$
t^{r / 2} \mathbb{E}\left(f\left(X_{t}\right) f^{\prime}\left(X_{t} / \sqrt{t}\right)\right) \rightarrow c^{r / 2} \mathbb{E}\left(|U|^{r} f^{\prime}(\sigma U)\right) .
$$

It would of course be possible to have estimates like (5.12) when $r \geq 1$ or $s>1$, but they are useless for us in this paper.

Proof. 1) First we prove a simple auxiliary result. We denote by $h$ the density of $\mathcal{N}(0,1)$, and $h_{p}(x)=x^{p} h(x)$ for $p \in \mathbb{N}$. Let $r \geq 0$ and $\alpha>0$ and $g$ be a nonnegative function such that $g(x) \leq|x|^{r}$ if $r>0$ and $g(x) \leq 1$ if $r=0$. For $p=0$ or $p=1$ we set

$$
k_{p, \alpha}(y)=\mathbb{E}\left(U^{p}\left(g(y+U) \bigwedge \alpha^{r}\right)\right)-\mathbb{E}\left(U^{p} g(U)\right) .
$$

By a change of variable we have

$$
k_{p, \alpha}(y)=\int\left(h_{p}(x-y)-h_{p}(x)\right) g(x) \mathrm{d} x-\int h_{p}(x-y)\left(g(x)-g(x) \bigwedge \alpha^{r}\right) \mathrm{d} x .
$$


The function $h_{p}$ is $C^{\infty}$, decreases exponentially fast as $|x| \rightarrow \infty$, and its two first derivatives are $h_{p}^{\prime}(x)=x^{p-1}(p-$ $\left.x^{2}\right) h(x)$ and $h_{p}^{\prime \prime}(x)=x^{p}\left(x^{2}-2 p-1\right) h(x)$ (recall $p=0$ or $p=1$ ). We also have $g(x)-g(x) \bigwedge \alpha^{r} \leq|x|^{r} 1_{\{|x|>\alpha\}}$. Hence the function $k_{p, \alpha}$ satisfies

$$
|y| \leq 1 \Rightarrow\left|k_{p, \alpha}(y)-y \int x^{p-1}\left(x^{2}-p\right) h(x) g(x) \mathrm{d} x\right| \leq C\left(y^{2}+\alpha^{-2}\right) .
$$

On the other hand $g(U+y) \leq C\left(|U|^{r}+\alpha^{r} \phi_{r}(y / \alpha)\right)$, hence $\left|k_{p, \alpha}(y)\right| \leq C\left(1+\alpha^{r} \phi_{r}(y / \alpha)\right)$. Putting all these facts together yields when $\alpha<\infty$ :

$$
\left|k_{p, \alpha}(y)\right| \leq\left\{\begin{array}{l}
C\left(\alpha^{-2}+\phi_{1}(y)+\alpha^{r} \phi_{r}(y / \alpha) 1_{\{|y|>1\}}\right) \\
C\left(\alpha^{-2}+\phi_{2}(y)+\alpha^{r} \phi_{r}(y / \alpha) 1_{\{|y|>1\}}\right)
\end{array} \quad \text { if } x \mapsto x^{p} g(x)\right. \text { is even. }
$$

2) Next we show that for (a) and (b) it is enough to prove the results when $f=\phi_{r}$. If $f \in \mathcal{E}_{r}^{\prime b}$, for any $\varepsilon>0$ we have $\left|f-\phi_{r}\right| \leq \varepsilon \phi_{r}$ on a neighborhood of the origin, whereas $f-\phi_{r}$ is bounded, so $\left|f-\phi_{r}\right|-\varepsilon \phi_{r}$ is smaller than a nonnegative continuous bounded function $g_{\varepsilon}$ vanishing on a neighborhood of 0 , therefore $g_{\varepsilon} \in \mathcal{E}_{2}^{\prime \prime \prime b} \cap C^{0}$ and $\left|f-\phi_{r}\right| \leq \varepsilon \phi_{r}+g_{\varepsilon}$ everywhere. Now, (5.8) yields $H_{t}\left(g_{\varepsilon}^{p}\right) \leq C_{\varepsilon, p} t$ for any $p \geq 1$, because $g_{\varepsilon}^{p} \in \mathcal{E}_{2}^{\prime \prime \prime b} \cap C^{0}$. Hence

$$
t^{-r / 2}\left|H_{t}(f)-H_{t}\left(\phi_{r}\right)\right| \leq \varepsilon t^{-r / 2} H_{t}\left(\phi_{r}\right)+C_{\varepsilon} t^{1-r / 2} .
$$

In the same way, by Hölder inequality and $\mathbb{E}\left(\left|X_{t}^{c}\right|^{q}\right) \leq C_{q} t^{q / 2}$ we get for $p \in(1,2 / r)$ :

$$
\begin{aligned}
t^{-1 / 2-r / 2}\left|\mathbb{E}\left(X_{t}^{c} f\left(X_{t}\right)\right)-\mathbb{E}\left(X_{t}^{c} \phi_{r}\left(X_{t}\right)\right)\right| & \leq C_{p} t^{-r / 2} H_{t}\left(\left|f-\phi_{r}\right|^{p}\right)^{1 / p} \\
& \leq C_{p} \varepsilon\left(t^{-r p / 2} H_{t}\left(\phi_{r p}\right)\right)^{1 / p}+C_{\varepsilon, p} t^{1 / p-r / 2} .
\end{aligned}
$$

These two estimates being true for all $\varepsilon>0$ we deduce that, if (5.11) holds for $f=\phi_{r}$ for all $r<2$, then it also holds for all $f \in \mathcal{E}_{r}^{\prime b}$ and all $r<2$ (recall $1<p<2 / r$, so $r p<2$ ).

Now let $r>0$ and assume that $X$ is continuous. Set $\phi_{q}^{\prime}(x)=|x|^{q}$. If $f \in \mathcal{E}_{r}^{\prime}$, for any $\varepsilon>0$ there is a $p_{\varepsilon}>r$ such that $\left|f-\phi_{r}\right| \leq \varepsilon \phi_{r}+C_{\varepsilon} \phi_{p_{\varepsilon}}^{\prime}$ (because $f$ has polynomial growth). Now we have $X_{t}=\sigma W_{t}+b t$, so obviously $H_{t}\left(\phi_{q}^{\prime}\right) \leq C_{q} t^{q / 2}$. Hence instead of (5.17) and (5.18) we get

$$
\begin{gathered}
t^{-r / 2}\left|H_{t}(f)-H_{t}\left(\phi_{r}\right)\right| \leq \varepsilon t^{-r / 2} H_{t}\left(\phi_{r}\right)+C_{\varepsilon} t^{p_{\varepsilon} / 2-r / 2}, \\
t^{-1 / 2-r / 2}\left|\mathbb{E}\left(X_{t}^{c} f\left(X_{t}\right)\right)-\mathbb{E}\left(X_{t}^{c} \phi_{r}\left(X_{t}\right)\right)\right| \leq C_{p} \varepsilon\left(t^{-r / 2} H_{t}\left(\phi_{r p}\right)\right)^{1 / p}+C_{\varepsilon, p} t^{p_{\varepsilon} / 2 p-r / 2} .
\end{gathered}
$$

Then as above, when $X$ is continuous we deduce that, if (5.11) holds for $f=\phi_{r}$ for all $r>0$, then it also holds for all $f \in \mathcal{E}_{r}^{\prime}$ and all $r>0$ (take $p$ such that $1<p<p_{\varepsilon} / r$ ).

Next we turn to (5.12). Il $f \in \mathcal{E}_{r}^{b}$ we have $\left|f-\phi_{r}\right| \leq g$ for some $g \in \mathcal{E}_{2}^{\prime \prime \prime b} \cap C^{0}$, whereas if $f \in \mathcal{E}_{r}$ we have the same majoration with $g=C \phi_{p}^{\prime}$ for some $p>r+2$. Then instead of (5.17) and (5.19) we get

$$
t^{-r / 2}\left|H_{t}(f)-H_{t}\left(\phi_{r}\right)\right| \leq \begin{cases}C t^{1-r / 2} & \text { if } r<2 \text { and } f \in \mathcal{E}_{r}^{b} \\ C t^{p / 2-r / 2} & \text { if } f \in \mathcal{E}_{r}^{b} \text { and } X \text { continuous. }\end{cases}
$$

Then clearly if (5.12) holds for $\phi_{r}$, it also holds for all $f \in \mathcal{E}_{r}^{b}$ or $f \in \mathcal{E}_{r}$, according to the case.

3) Now we prove (a) and (b) for $f=\phi_{r}$. Set $\beta_{0}(t)=t^{-r / 2} H_{t}\left(\phi_{r}\right)-c^{r / 2} m_{r}$ and $\beta_{1}(t)=t^{-r} \mathbb{E}\left(X_{t}^{c} \phi_{r}\left(X_{t}\right)\right)$. Recall that the pair $\left(X_{t}, X_{t}^{c}\right)$ has the same law as $\left(\sigma U \sqrt{t}+X_{t}^{\prime \prime}, \sigma U \sqrt{t}\right)$, with $U$ independent of $X_{t}^{\prime \prime}$. Then we 
get for $p=0$ or $p=1$ :

$$
\begin{aligned}
\beta_{p}(t) & =\sigma^{p+r}\left(\mathbb{E}\left(U^{p}\left(\left|U+X_{t}^{\prime \prime} / \sigma \sqrt{t}\right|^{r} \bigwedge(c t)^{-r / 2}\right)\right)-\mathbb{E}\left(U^{p}|U|^{r}\right)\right) \\
& =\sigma^{p+r} \mathbb{E}\left(k_{p, 1 / \sigma \sqrt{t}}\left(X_{t}^{\prime \prime} / \sigma \sqrt{t}\right)\right),
\end{aligned}
$$

where $k_{p, \alpha}$ is associated with function $g(x)=|x|^{r}$. The first estimate in (5.16) yields

$$
\left|\beta_{p}(t)\right| \leq C\left(t+\mathbb{E}\left(\phi_{2}\left(X_{t}^{\prime \prime} / \sigma \sqrt{t}\right)\right)^{1 / 2}+t^{-r / 2} \mathbb{E}\left(\phi_{r}\left(X_{t}^{\prime \prime}\right)\right)\right) .
$$

Then $\beta_{p}(t) \rightarrow 0$, that is (5.11), follows from (4.2) and from (5.9) applied to $X^{\prime \prime}$ and with $s=2$ in general, and from the property $X_{t}^{\prime \prime}=b t$ when $X$ is continuous. Next, the second estimate in (5.16) yields, since $g$ is even:

$$
\left|\beta_{0}(t)\right| \leq C\left(t+\mathbb{E}\left(\phi_{2}\left(X_{t}^{\prime \prime} / \sigma \sqrt{t}\right)\right)+t^{-r / 2} \mathbb{E}\left(\phi_{r}\left(X_{t}^{\prime \prime}\right) 1_{\left\{\left|X_{t}^{\prime \prime}\right|>\sigma \sqrt{t}\right\}}\right)\right) .
$$

Therefore (5.12) when $r<1$ readily follows from (4.2) and (5.10). Finally if $X$ is continuous, $X_{t}^{\prime \prime}=b t$, so (5.20) gives $\left|\beta_{0}(t)\right| \leq C t$ as soon as $|b t| \leq \sigma \sqrt{t}$, hence the first estimate in (5.12).

4) Now let $f$ be bounded. Exactly as above, we get for $p=0$ or $p=1$ :

$$
\mathbb{E}\left(\left(X_{t}^{c}\right)^{p} f\left(X_{t} / \sqrt{t}\right)\right)-t^{p / 2} \mathbb{E}\left((\sigma U)^{p} f(\sigma U)\right)=t^{p / 2} c^{p / 2} \mathbb{E}\left(k_{p, \infty}\left(X^{\prime \prime} / \sqrt{t}\right)\right),
$$

where $k_{p, \infty}$ is associated with the bounded function $g(x)=f(\sigma x)$ and $\alpha=\infty$. Here $k_{p, \infty}$ is bounded, so instead of (5.16) we have $\left|k_{p, \infty}(y)\right| \leq C \phi_{1}(y)$ in general, and also $\left|k_{0, \infty}(y)\right| \leq C \phi_{2}(y)$ if further $f$ is even. Then we conclude (5.13) and (5.14) like in Step 3, by using (4.2). This gives (c), and (d) is proved exactly as (5.11), except that we take $g(x)=|x|^{r} f^{\prime}(\sigma x)$.

Lemma 5.3. Assume $c=0$ and $1 \in I$, and let $f \in \mathcal{E}_{r}^{\prime b}$ for some $r<1$. Then

$$
t^{-r} H_{t}(f) \rightarrow|\bar{b}|^{r}
$$

as $t \rightarrow 0$. Moreover we have

$$
\begin{gathered}
r \in I, \quad f \in \mathcal{E}_{r}^{b} \cap C^{0, F} \quad \Rightarrow \quad \frac{1}{t}\left(H_{t}(f)-t^{r}|\bar{b}|^{r}\right) \rightarrow F(f) . \\
2 r \in I, \quad f \in \mathcal{E}_{r}^{b} \quad \Rightarrow \quad \frac{1}{\sqrt{t}}\left(H_{t}(f)-t^{r}|\bar{b}|^{r}\right) \rightarrow 0 .
\end{gathered}
$$

Proof. If $H_{t}^{\prime}(f)=\mathbb{E}\left(f\left(X_{t}^{\prime}\right)\right)$, we can apply (5.9) to the process $X^{\prime}$, whose drift $\bar{b}^{\prime}$ vanishes and whose associated set $I^{\prime}$ is equal to $I$. This gives

$$
\left.\begin{array}{ll}
H_{t}^{\prime}\left(\phi_{r}\right)=\mathrm{o}\left(t^{r}\right) & (\text { apply (5.9) with } s=1) \\
2 r \in I \Rightarrow H_{t}^{\prime}\left(\phi_{r}\right)=\mathrm{o}\left(t^{1 / 2}\right) & (\text { apply (5.9) with } s=2 r)
\end{array}\right\} .
$$

Since $X_{t}=\bar{b} t+X_{t}^{\prime}$ and ||$x+\left.y\right|^{r}-\left.|x|^{r}|\leq| y\right|^{r}$, it is easy to check that $A_{t}=\phi_{r}\left(X_{t}\right)-|\bar{b} t|^{r} \mid-\phi_{r}\left(X_{t}^{\prime}\right)$ satisfies $\left|A_{t}\right| \leq 2|\bar{b} t|^{r}$ and also $\left|A_{t}\right| \leq 2 \phi_{r}\left(X_{t}^{\prime}\right)$.

First we deduce $\left.\left|\phi_{r}\left(X_{t}\right)-\right| \bar{b} t\right|^{r} \mid \leq 3 \phi_{r}\left(X_{t}^{\prime}\right)$, hence $\left.\left|H_{t}\left(\phi_{r}\right)-t^{r}\right| \bar{b}\right|^{r} \mid \leq 3 H_{t}^{\prime}\left(\phi_{r}\right)$, so (5.21) and (5.23) for $f=\phi_{r}$ follow from (5.24). If $f \in \mathcal{E}_{r}^{\prime b}$ then (5.17) holds and we deduce (5.21) for this $f$. If $f \in \mathcal{E}_{r}^{b}$ then $\left|f-\phi_{r}\right| \in \mathcal{E}_{2}^{\prime \prime \prime b}$, so (5.23) for $\phi_{r}$ and (5.8) imply (5.23) for $f$. 
Next, with the notation (5.1) we have $\left|A_{t}\right| \leq 2\left(\phi_{r} \psi_{\eta}\right)\left(X_{t}^{\prime}\right)+2|\bar{b} t|^{r} 1_{\left\{\left|X_{t}^{\prime}\right|>\eta\right\}}$, and thus also $\left|A_{t}\right| \leq 2\left(\phi_{r} \psi_{\eta}\right)\left(X_{t}^{\prime}\right)+$ $C_{\eta} t^{r} \phi_{2}\left(X_{t}^{\prime}\right)$. Therefore

$$
\left.\left|H_{t}\left(\phi_{r}\right)-t^{r}\right| \bar{b}\right|^{r}-H_{t}^{\prime}\left(\phi_{r}\right) \mid \leq 2 H_{t}^{\prime}\left(\phi_{r} \psi_{\eta}\right)+C_{\eta} t^{r} H_{t}^{\prime}\left(\phi_{2}\right) .
$$

If $r \in I$, then (5.8) applied to $X^{\prime}$ yields that $\frac{1}{t} H_{t}^{\prime}\left(\phi_{r}\right) \rightarrow F\left(\phi_{r}\right)$, and also $\frac{1}{t} H_{t}^{\prime}\left(\phi_{r} \psi_{\eta}\right) \rightarrow F\left(\phi_{r} \psi_{\eta}\right)$ and $\frac{1}{t} H_{t}^{\prime}\left(\phi_{2}\right) \rightarrow F\left(\phi_{2}\right)$. We deduce that

$$
\limsup \left|\frac{1}{t}\left(H_{t}\left(\phi_{r}\right)-t^{r}|\bar{b}|^{r}\right) F\left(\phi_{r}\right)\right| \leq 2 F\left(\phi_{r} \psi_{\eta}\right)
$$

and, since $F\left(\phi_{r} \psi_{\eta}\right) \rightarrow 0$ as $\eta \rightarrow 0$, we deduce (5.22) for $f=\Phi_{r}$. Finally if $f \in \mathcal{E}_{r} \cap C^{0, F}$, then $f-\phi_{r} \in \mathcal{E}_{2}^{\prime \prime \prime b} \cap C^{0, F}$, so (5.8) yields $\frac{1}{t} H_{t}\left(f-\phi_{r}\right) \rightarrow F\left(f-\phi_{r}\right)$, hence (5.22) also holds for $f$.

Lemma 5.4. Either we are in one of the cases described in Theorem 2.1-(ii) and every $f \in \mathcal{E}_{r}^{\prime b}$ satisfies $\sup _{t} \frac{1}{t}\left|H_{t}(f)\right|<\infty$, or $\frac{1}{t} H_{t}\left(\phi_{r}\right) \rightarrow \infty$ and $H_{t}(f) / H_{t}\left(\phi_{r}\right) \rightarrow 1$ for all $f \in \mathcal{E}_{r}^{\prime b}$.

Proof. Everything is a simple consequence of (5.8), (5.11) and (5.21)).

\section{Lemma 5.5.}

a) If either $f \in \mathcal{E}_{1}^{\prime \prime \prime}$, or $f \in \mathcal{E}_{r}^{b}$ for some $r<1$ with $2 r \in I$ and $c=0$, the family $\frac{1}{t} \Gamma_{t}(f)$ is bounded (recall (2.3) for $\Gamma_{t}(f)$ ), and it converges to $F\left(f^{2}\right)$ if further $f \in C^{0, F}$.

b) If $r=1$ the family $\frac{1}{t} \Gamma_{t}(f)$ is bounded for all $f \in \mathcal{E}_{r}^{\prime b}$, and it converges to $F\left(f^{2}\right)+c\left(1-m_{1}^{2}\right)$ if further $f \in C^{0, F}$.

c) In all other cases (that is $2 r \notin I$, or $r<1$ and $c>0$ ), we have $\frac{1}{t} \Gamma_{t}\left(\phi_{r}\right) \rightarrow \infty$ and $\Gamma_{t}(f) / \Gamma_{t}\left(\phi_{r}\right) \rightarrow 1$ for all $f \in \mathcal{E}_{r}^{b}$.

Proof. (b) and the case $f \in \mathcal{E}_{1}^{\prime \prime \prime b}$ of (a) and the case $c>0$ of (c) are trivial consequences of Lemma 5.2 and of (5.8).

It remains to study the case $r<1$ and $c=0$. Let $f \in \mathcal{E}_{r}^{b}$, so $f=\phi_{r}$ around 0 . Let also $p=1$ or $p=2$. Since $f^{p}-\phi_{r}^{p} \in \mathcal{E}_{2}^{\prime \prime b}$, the family $\frac{1}{t} H_{t}\left(f^{p}-\phi_{r}^{p}\right)$ is bounded, and converges to $F\left(f^{p}-\phi_{r}^{p}\right)$ if further $f$ is $F$-a.e. continuous (apply (5.8)). Moreover (5.9) applied with $s=2$ yields $H_{t}\left(f+\phi_{r}\right) \leq C H_{t}\left(\phi_{r}\right)=\mathrm{o}\left(t^{r / 2}\right)$. Since

$$
\frac{1}{t}\left(\Gamma_{t}(f)-\Gamma_{t}\left(\phi_{r}\right)\right)=\frac{1}{t} H_{t}\left(f^{2}-\phi_{r}^{2}\right)-\frac{1}{t} H_{t}\left(f-\phi_{r}\right) H_{t}\left(f+\phi_{r}\right),
$$

we deduce that the family $\frac{1}{t}\left(\Gamma_{t}(f)-\Gamma_{t}\left(\phi_{r}\right)\right)$ is bounded, and converges to $F\left(f^{2}-\phi_{r}^{2}\right)$ if further $f$ is $F$-a.e. continuous. We deduce the following properties for any $f \mathcal{E}_{r}$ :

$$
\begin{array}{ll}
\frac{1}{t} \Gamma_{t}\left(\phi_{r}\right) \rightarrow F\left(\phi_{r}^{2}\right)<\infty & \Rightarrow\left\{\begin{array}{l}
\frac{1}{t} \Gamma_{t}(f) \rightarrow F\left(f^{2}\right) \\
\sup _{t}\left|\frac{1}{t} \Gamma_{t}(f)\right|<\infty
\end{array} \text { if further } f \in C^{0, F}\right. \\
\frac{1}{t} \Gamma_{t}\left(\phi_{r}\right) \rightarrow \infty & \Rightarrow\left\{\begin{array}{l}
\frac{1}{t} \Gamma_{t}(f) \rightarrow \infty \\
\Gamma_{t}(f) / \Gamma_{t}\left(\phi_{r}\right) \rightarrow 1 .
\end{array}\right\}
\end{array}
$$

Suppose now that $2 r \notin I$. Define $\psi_{\eta}$ by (5.1), so that $\frac{1}{t}\left(\Gamma_{t}\left(\phi_{r}\right)-\Gamma_{t}\left(\phi_{r} \psi_{\eta}\right)\right) \rightarrow F\left(\phi_{r}^{2}-\left(\phi_{r} \psi_{\eta}\right)^{2}\right)$ by what precedes. Since and $\Gamma_{t}\left(\phi_{r} \psi_{\eta}\right) \geq 0$, we get

$$
\liminf _{t \rightarrow 0} \frac{1}{t} \Gamma_{t}\left(\phi_{r}\right) \geq F\left(\phi_{r}^{2}\left(1-\psi_{\eta}^{2}\right)\right)
$$

and since $F\left(\phi_{r}^{2}\right)=\infty$ by hypothesis and $1-\psi_{\eta}^{2}$ increases to 1 as $\eta \rightarrow 0$, we obtain $\frac{1}{t} \Gamma_{t}\left(\phi_{r}\right) \rightarrow \infty$ : this prove (c) in the case $c=0$.

Finally we suppose that $r<1$ and $2 r \in I$ and $c=0$. In order to get the remaining part of (a) it suffices to show $\frac{1}{t} \Gamma_{t}\left(\phi_{r}\right) \rightarrow F\left(\phi_{r}^{2}\right)$. We single out the following three cases: 
1) Assume either $r>1 / 2$, or $r \leq 1 / 2$ and $\bar{b}=0$. Then (5.8) yields $\frac{1}{t} H_{t}\left(\phi_{r}^{2}\right) \rightarrow F\left(\phi_{r}^{2}\right)$, and (5.9) applied with $s=2 r$ yields $H_{t}\left(\phi_{r}\right)=\mathrm{o}(\sqrt{t})$, hence the result is obvious.

2) Assume $r=1 / 2$ and $\bar{b} \neq 0$. (5.9) yields $\frac{1}{t} H_{t}\left(\phi_{r}^{2}\right) \rightarrow F\left(\phi_{r}^{2}\right)+|\bar{b}|$, and (5.21) gives $\frac{1}{\sqrt{t}} H_{t}\left(\phi_{r}\right) \rightarrow \sqrt{|\bar{b}|}$, so we obtain the result.

3) Assume $r<1 / 2$ and $\bar{b} \neq 0$. Then (5.22) and (5.23) yield

$$
\frac{1}{t} H_{t}\left(\phi_{r}^{2}\right)=t^{2 r-1}|\bar{b}|^{2 r}+F\left(\phi_{r}^{2}\right)+o(1), \quad \frac{1}{\sqrt{t}} H_{t}\left(\phi_{r}\right)=t^{r-1 / 2}|\bar{b}|^{r}+o(1)
$$

and the result follows.

\subsection{Proof of Theorem 2.1-(iii)}

We suppose that we are not in the cases of (ii) of this theorem. The first claim of (iii) follows from Lemma 5.4. For the second claim, we let $f \in \mathcal{E}_{r}^{\prime}$, so for any $\varepsilon>0$ there is $g_{\varepsilon} \in \mathcal{E}_{2}^{\prime \prime \prime} \cap C^{0}$ such that $\left|f-\phi_{r}\right| \leq \varepsilon \phi_{r}+g_{\varepsilon}$. Furthermore, we know that $\frac{\Delta_{n}}{H_{\Delta_{n}}\left(\phi_{r}\right)} V^{n}\left(g_{\varepsilon}\right) \stackrel{\text { u.c.p. }}{\longrightarrow} 0$ by (i) of the theorem and the property $H_{\Delta_{n}}\left(\phi_{r}\right) / \Delta_{n} \rightarrow \infty$. So it is enough to prove the claim when $f=\phi_{r}$.

So it is indeed enough to prove that $Y_{t}^{n}:=\frac{\Delta_{n}}{H_{\Delta_{n}}\left(\phi_{r}\right)} V^{n}\left(\phi_{r}\right)_{t}-\Delta_{n}\left[t / \Delta_{n}\right] \stackrel{\text { u.c.p. }}{\longrightarrow} 0$. Note that $Y_{t}^{n}$ is the sum of $\left[t / \Delta_{n}\right]$ i.i.d. variables distributed as $\zeta_{n}=\Delta_{n}\left(\phi_{r}\left(\Delta_{i}^{n} X\right) / H_{\Delta_{n}}\left(\phi_{r}\right)-1\right)$. Then $\mathbb{E}\left(\zeta_{n}\right)=0$ and

$$
\mathbb{E}\left(\zeta_{n}^{2}\right)=\frac{\Delta_{n}^{2} \Gamma_{\Delta_{n}}\left(\phi_{r}\right)}{H_{\Delta_{n}}\left(\phi_{r}\right)^{2}} \leq \frac{\Delta_{n}^{2} H_{\Delta_{n}}\left(\phi_{r}^{2}\right)}{H_{\Delta_{n}}\left(\phi_{r}\right)^{2}} \leq C \frac{\Delta_{n}^{2}}{H_{\Delta_{n}}\left(\phi_{r}\right)}
$$

because $\phi_{r} \leq 1$. Hence $E\left(\zeta_{n}^{2}\right) / \Delta_{n} \rightarrow 0$, and $Y^{n} \stackrel{\text { u.c.p. }}{\longrightarrow} 0$ readily follows.

\section{Proofs of the other theorems about $V^{n}(f)$ And $V^{\prime n}(f)$}

\subsection{Proof of Theorem $\mathbf{2 . 2}$}

(i) It is enough to prove that the processes $Y_{t}^{n}=\Delta_{n}^{1-r / 2} V^{n}(f)_{t}-\Delta_{n} c^{r / 2} m_{r}\left[t / \Delta_{n}\right]$ converge u.c.p. to 0 . Now $Y_{t}^{n}$ is the sum of $\left[t / \Delta_{n}\right]$ i.i.d. variables, all distributed as $\zeta_{n}=\Delta_{n}\left(\Delta_{n}^{-r / 2} f\left(\Delta_{i}^{n} X\right)-c^{r / 2} m_{r}\right)$. If $f \in \mathcal{E}_{r}^{\prime b}$ and $r \in(0,2)$, or if $f \in \mathcal{E}_{r}^{\prime}$ and $r \in[2, \infty)$ when there is no jump, we have

$$
\mathbb{E}\left(\zeta_{n}\right)=\Delta_{n}\left(\Delta_{n}^{-r / 2} H_{\Delta_{n}}(f)-c^{r / 2} m_{r}\right), \quad \mathbb{E}\left(\zeta_{n}^{2}\right) \leq \Delta_{n}^{2-r} H_{\Delta_{n}}\left(f^{2}\right) .
$$

Then (5.11), and also (5.8) when $r \geq 1$, yield $E\left(\zeta_{n}\right) / \Delta_{n} \rightarrow 0$ and $E\left(\zeta_{n}^{2}\right) / \Delta_{n} \rightarrow 0$, and $Y^{n} \stackrel{\text { u.c.p. }}{\longrightarrow} 0$ readily follows.

It remains to study the case $f \in \mathcal{E}_{r}^{\prime} \backslash \mathcal{E}_{r}^{\prime b}$ when $r<2$. We have $|f-g| \leq h$ for some $g \in \mathcal{E}_{r}^{\prime b}$ and some $h \in \mathcal{E}_{2}^{\prime \prime \prime} \cap C^{0}$, and Theorem 2.1-(i) yields $\Delta_{n}^{1-r / 2} V^{n}(h) \stackrel{\text { u.c.p. }}{\longrightarrow} 0$ (recall $r<2$ ); since (i) holds for $g$ it also holds for $f$.

(ii) Let $f$ be bounded. We do as above with $Y_{t}^{n}=\Delta_{n} V^{\prime n}(f)_{t}-\Delta_{n} \mathbb{E}(f(\sigma U))\left[t / \Delta_{n}\right]$ and $\zeta_{n}=\Delta_{n}\left(f\left(\Delta_{i}^{n} X / \sqrt{\Delta_{n}}\right)-\right.$ $\mathbb{E}(f(\sigma U)))$. We have

$$
\mathbb{E}\left(\zeta_{n}\right)=\Delta_{n}\left(\mathbb{E}\left(f\left(\Delta_{i}^{n} X / \sqrt{\Delta_{n}}\right)\right)-\mathbb{E}(f(\sigma U))\right), \quad \mathbb{E}\left(\zeta_{n}^{2}\right) \leq \Delta_{n}^{2} \mathbb{E}\left(f\left(\Delta_{i}^{n} X / \sqrt{\Delta_{n}}\right)^{2}\right)
$$

Then (5.13) yields $\left|E\left(\zeta_{n}\right)\right| / \Delta_{n} \rightarrow 0$ and $E\left(\zeta_{n}^{2}\right) / \Delta_{n} \rightarrow 0$, and $Y^{n} \stackrel{\text { u.c.p. }}{\longrightarrow} 0$ follows. 


\subsection{Proof of Theorems 2.3 and 2.4-(ii)}

Step 1. Let $f \in \mathcal{E}_{r} \cap C^{0, F}$ with either $1 \leq r<2$, or $0<r<1$ and $2 r \in I$ and $c=0$. Let $\varphi$ be continuous with $1_{[-a, a]} \leq \varphi \leq 1_{\left[-a^{\prime}, a^{\prime}\right]}$ for some $0<a<a^{\prime}<\infty$, or $\varphi \equiv 1$ when $f$ is bounded, so the process $\Sigma(f, \varphi)$ is well defined. For simplicity we always write $v_{n}=H_{\Delta_{n}}(f \varphi)$. We want to prove that $V^{n}(f)_{t}-v_{n} t$ converges stably in law to $Z=\Sigma(f, \varphi)+W^{\prime}$ with $W^{\prime}$ a Wiener process independent of $X$ and with unit variance $c\left(m_{2}-m_{1}^{2}\right)$ when $r=1$ and $c>0$, and $W^{\prime}=0$ otherwise (hence we get $V^{n}(f)_{t}-v_{n} t \stackrel{\mathbb{P}}{\longrightarrow} \Sigma(f, \varphi)$ ).

Suppose for a moment that the result holds whenever $f$ is bounded. Then if we start with an unbounded $f$, we set $f_{\eta}=f \psi_{\eta}$ (recall (5.1)). Clearly $f_{\eta} \varphi=f \varphi$ as soon as $\eta>a^{\prime}$, so for such $\eta$ we have on the one hand that $V^{n}\left(f_{\eta}\right)_{t}-v_{n} t$ converges stably in law to $\Sigma\left(f_{\eta}, \varphi\right)+W^{\prime}$, and on the other hand $V^{n}\left(f-f_{\eta}\right) \stackrel{\mathbb{P}}{\longrightarrow}\left(f-f_{\eta}\right) \star \mu$ by Theorem 2.1-(i). Since further $\Sigma\left(f-f_{\eta}, \varphi\right)$ and $\left(f-f_{\eta}\right) \star \mu$ both go to 0 locally uniformly (pointwise in $\left.\omega\right)$, it is clear that $V^{n}(f)_{t}-v_{n} t$ converges stably in law to $\Sigma(f, \varphi)+W^{\prime}$.

Step 2. In the sequel we suppose $f$ bounded. Observe that $v_{n}=\mathbb{E}\left((f \varphi)\left(X_{\Delta_{n}}\right)\right) \rightarrow 0$ because $f \varphi$ is bounded and $(f \varphi)(x) \rightarrow 0$ as $x \rightarrow 0$ and $X_{\Delta_{n}} \rightarrow 0$. Hence it is enough to show the convergence of the processes $Z_{t}^{n}=V^{n}(f)_{t}-v_{n}\left[t / \Delta_{n}\right]$ to $Z^{\prime}=\Sigma(f, \varphi)$.

Now, $Z_{t}^{n}$ is the sum of $\left[t / \Delta_{n}\right]$ i.i.d. variables distributed as $\chi_{n}=f\left(X_{\Delta_{n}}\right)-v_{n}$. Also, the characteristics of $Z^{\prime}$ are $\left(b^{\prime}, 0, F^{\prime}\right)$ where $b^{\prime}=\int f(x)\left(1_{\{|f(x)| \leq 1\}}-\varphi(x)\right) F(\mathrm{~d} x)$ and $F^{\prime}$ is the image of $F$ by $f$. Then in view of Lemma 4.3 it is enough to prove the following properties, for some $C_{b}^{1}$ truncation function $\psi$ such that $\psi(x)=x$ when $|x| \leq 2 \sup _{y}|f(y)|$ and with $c^{\prime}=0$ when $r \neq 1$ and $c^{\prime}=c\left(1-m_{1}^{2}\right)$ when $r=1$ :

$$
\begin{aligned}
\frac{1}{\Delta_{n}} \mathbb{E}\left(\psi\left(\chi_{n}\right)\right) & \rightarrow F(f(1-\varphi)), \\
\frac{1}{\Delta_{n}} \mathbb{E}\left(\psi\left(\chi_{n}\right)^{2}\right) & \rightarrow c^{\prime}+F\left(f^{2}\right), \\
\frac{1}{\Delta_{n}} \mathbb{E}\left(\left|\chi_{n}-Z_{\Delta_{n}}^{\prime}\right|>\varepsilon\right) & \rightarrow 0 \quad \forall \varepsilon>0,
\end{aligned}
$$

plus the following, when $r=1$ and $c>0$ :

$$
\frac{1}{\Delta_{n}} \mathbb{P}\left(\psi\left(\chi_{n}\right) X_{\Delta_{n}}^{c}\right) \rightarrow 0
$$

Step 3. By our choice of $\psi$, we have $\psi\left(\chi_{n}\right)=f\left(X_{\Delta_{n}}\right)-v_{n}$. Then the left side of $(6.1)$ is $\frac{1}{\Delta_{n}} H_{\Delta_{n}}(f(1-\varphi))$. Since $f(1-\varphi) \in \mathcal{E}_{2}^{\prime \prime b} \cap C_{r}^{0, F},(5.8)$ yields (6.1). Also, the left side of $(6.2)$ is $\frac{1}{\Delta_{n}}\left(\Gamma_{\Delta_{n}}(f)+H_{\Delta_{n}}(f(1-\varphi))^{2}\right)$, which by what precedes has the same limit as $\frac{1}{\Delta_{n}} \Gamma_{\Delta_{n}}(f)$. Then Lemma 5.5-(a,b) gives (6.2). Finally, the left side of (6.4) is $\frac{1}{\Delta_{n}} \mathbb{E}\left(X_{\Delta_{n}}^{c} f\left(X_{\Delta_{n}}\right)\right)$, hence (6.4) when $r=1$ and $c>0$ follows from (5.11).

Step 4. It remains to prove (6.3). Let $\varepsilon \in(0,1)$ be such that $f(x)=|x|^{r}$ for $|x| \leq \varepsilon$, and use the notation $X(\varepsilon), S_{p}, R_{p}$ given around (5.3), and also $Z_{t}^{\prime \prime}=Z_{t}^{\prime}-\sum_{s \leq t} \Delta Z_{s}^{\prime} 1_{\left\{\left|\Delta X_{s}\right|>\varepsilon\right\}}$. We divide the sample space into three sets: $B_{n}=\left\{S_{1}>\Delta_{n}\right\}, B_{n}^{\prime}=\left\{S_{1} \leq \Delta_{n}<S_{2}\right\}$, and the complement $B_{n}^{\prime \prime}=\left\{S_{2} \leq \Delta_{n}\right\}$. Observe that

$$
\chi_{n}-Z_{\Delta_{n}}^{\prime}= \begin{cases}f\left(X(\varepsilon)_{\Delta_{n}}\right)-v_{n}-Z_{\Delta_{n}}^{\prime \prime} & \text { on } B_{n} \\ f\left(X(\varepsilon)_{\Delta_{n}}+R_{1}\right)-v_{n}-Z_{\Delta_{n}}^{\prime \prime}-f\left(R_{1}\right) & \text { on } B_{n}^{\prime}\end{cases}
$$

and $B_{n}^{\prime}$ is independent of $\left(X(\varepsilon), Z^{\prime \prime}, R_{1}\right)$ and $\mathbb{P}\left(B_{n}^{\prime}\right)=\mathrm{O}\left(\Delta_{n}\right)$ and $\mathbb{P}\left(B_{n}^{\prime \prime}\right)=\mathrm{O}\left(\Delta_{n}^{2}\right)$, so

$$
\begin{gathered}
\frac{1}{\Delta_{n}} \mathbb{P}\left(\left|\chi_{n}-Z_{\Delta_{n}}^{\prime}\right|>3 \varepsilon^{r}\right) \leq \frac{1}{\Delta_{n}} \mathbb{P}\left(\left|f\left(X^{\prime}(\varepsilon)_{\Delta_{n}}\right)-v_{n}-Z_{\Delta_{n}}^{\prime \prime}\right|>3 \varepsilon^{r}\right) \\
+C \mathbb{P}\left(\left|f\left(X(\varepsilon)_{\Delta_{n}}+R_{1}\right)-v_{n}-Z_{\Delta_{n}}^{\prime \prime}-f\left(R_{1}\right)\right|>3 \varepsilon^{r}\right)+C \Delta_{n} .
\end{gathered}
$$


Since $X(\varepsilon)$ (resp. $\left.Z^{\prime \prime}\right)$ is a Lévy process whose Lévy measure charges only the interval $(-\varepsilon, \varepsilon)$ (resp. $\left.\left(0, \varepsilon^{r}\right)\right),(5.8)$ yields $\frac{1}{\Delta_{n}} \mathbb{P}\left(\left|X(\varepsilon)_{\Delta_{n}}\right|>\varepsilon\right) \rightarrow 0$ and $\frac{1}{\Delta_{n}} \mathbb{P}\left(\left|Z_{\Delta_{n}}^{\prime \prime}\right|>\varepsilon^{r}\right) \rightarrow 0$, whereas $v_{n} \rightarrow 0$ : hence the first term on the right side above goes to 0 . We also have $X(\varepsilon)_{\Delta_{n}} \rightarrow 0$ and $Z_{\Delta_{n}}^{\prime \prime} \rightarrow 0$, and for almost all $\omega$ the function $f$ is continuous at $R_{1}(\omega)$, hence the second term on the right side above goes to 0 , as well as the third one of course. Then (6.3) holds, and we are finished.

\subsection{Proof of Theorem 2.4-(i)}

Let $f \in \mathcal{E}_{r}$ for some $r<1$, and assume $c=0$ and $2 r \notin I$. Then Lemma 5.5-(c) yields that $u_{n}=$ $\sqrt{\Delta_{n} / \Gamma_{\Delta_{n}}\left(\phi_{r}\right)}$ goes to 0 .

There is $h \in \mathcal{E}_{2}^{\prime \prime \prime} \cap C^{0}$ such that $\left|f-\phi_{r}\right| \leq h$, and $\left|V^{n}(f)-V^{n}\left(\phi_{r}\right)\right| \leq V^{n}(h)$ and by Theorem 2.1-(i) we know that $V^{n}(h) \stackrel{\mathbb{P}}{\longrightarrow} \Sigma(h)$. Since $u_{n} \rightarrow 0$, it is then obvious that we only need to prove the result for $f=\phi_{r}$. We also have $\left|u_{n} H_{\Delta_{n}}\left(\phi_{r}\right)\right| \leq u_{n} \rightarrow 0$, so it is enough to prove the convergence of the processes $Y_{t}^{n}=u_{n}\left(V^{n}(f)_{t}-H_{\Delta_{n}}(f)\left[t / \Delta_{n}\right]\right)$.

Note that $Y_{t}^{n}$ is the sum of $\left[t / \Delta_{n}\right]$ i.i.d. variables distributed as $\chi_{n}=u_{n}\left(f\left(X_{\Delta_{n}}\right)-H_{\Delta_{n}}(f)\right)$. Moreover $X^{c}=0$, and $\left|\chi_{n}\right| \leq u_{n} \rightarrow 0$, so in view of Lemma 4.3 it is enough to prove that $\frac{1}{\Delta_{n}} \mathbb{E}\left(\chi_{n}\right) \rightarrow 0$ and $\frac{1}{\Delta_{n}} \mathbb{E}\left(\chi_{n}^{2}\right) \rightarrow 1$. But $\Gamma_{\Delta_{n}}(f)$ is the variance of $f\left(X_{\Delta_{n}}\right)$, so $\mathbb{E}\left(\chi_{n}\right)=0$ and $\mathbb{E}\left(\chi_{n}^{2}\right)=\Delta_{n}$, and we are done.

\subsection{Proof of Theorem 2.5}

(i-1) We first prove the result when $f \in \mathcal{E}_{r}$ for some $r<1$. We have $\left|f-\phi_{r}\right| \leq g$ for some $g_{\varepsilon} \in \mathcal{E}_{2}^{\prime \prime} \cap C^{0}$, and Theorem 2.1-(i) implies that $V^{n}\left(g_{\varepsilon}\right)$ converges to a finite-valued limiting process, so because $r<1$ it is clearly enough to prove the result for $f=\phi_{r}$. Since $\Delta_{n}^{1 / 2-r / 2} H_{\Delta_{n}}\left(\phi_{r}\right) \rightarrow 0$ by (5.11), we can replace $t$ by $\Delta_{n}\left[t / \Delta_{n}\right]$ in the centering term of our pre-limiting processes. Therefore we are left to proving the stable convergence in law of processes which at time $t$ are sums of $\left[t / \Delta_{n}\right]$ i.i.d. variables distributed as $\chi_{n}=u_{n}\left(\zeta_{n}-\mathbb{E}\left(\zeta_{n}\right)\right)$, where $u_{n}=\Delta_{n}^{1 / 2-r / 2}$ and $\zeta_{n}=\phi_{r}\left(X_{\Delta_{n}}\right)$.

Note that $u_{n} \rightarrow 0$ and $\phi_{r}$ is bounded. Then, choosing an adequate truncation function $\psi$, and since $\chi_{n}$ has mean 0 , we will deduce the result from Lemma 4.3, provided we prove

$$
\begin{gathered}
\frac{1}{\Delta_{n}} u_{n}^{2}\left(\mathbb{E}\left(\zeta_{n}^{2}\right)-\mathbb{E}\left(\zeta_{n}\right)^{2}\right) \rightarrow c^{r}\left(m_{2 r}-m_{r}^{2}\right), \\
\frac{1}{\Delta_{n}} u_{n} \mathbb{E}\left(X_{\Delta_{n}}^{c} \zeta_{n}\right) \rightarrow 0 .
\end{gathered}
$$

These two properties follow from (5.11) (because $2 r<2$ here).

Now we turn to the case without jump, with $f \in \mathcal{E}_{r}$ and $r \geq 1$. In view of the last statement of Lemma 5.2-(a), we obviously can replace in the centering term $t \Delta_{n}^{-r / 2} H_{\Delta_{n}}\left(\phi_{r}\right)$ by $t c^{r / 2} m_{r}$, and also by $\left[t / \Delta_{n}\right] \Delta_{n}^{1-r / 2} H_{\Delta_{n}}(f)$. With this last version for centering, we are exactly in the same situation than above, with $u_{n}$ and $\chi_{n}$ similar, but $\zeta_{n}=f\left(X_{\Delta_{n}}\right)$. We have to prove (6.5) and (6.6), which again hold because of (5.11). However, there is a difference here, namely $u_{n}$ no longer tends to 0 . So additionally to the previous properties we have to prove the following Lindeberg condition:

$$
\frac{1}{\Delta_{n}} u_{n}^{2} \mathbb{E}\left(\zeta_{n}^{2} 1_{\left\{\left|u_{n} \zeta_{n}\right|>\varepsilon\right\}}\right) \rightarrow 0 \quad \forall \varepsilon>0
$$

Since $\left|\zeta_{n}\right| \leq C\left(\Delta_{n}^{r}+\left|X_{\Delta_{n}}^{c}\right|^{r}\right)$ has the same law as $C\left(\Delta_{n}^{r}+\Delta_{n}^{r / 2}|U|^{r}\right)$, this Lindeberg condition is obvious and we have the desired convergence.

(ii-1) Since $f$ is bounded, we clearly can replace $t$ by $\Delta_{n}\left[t / \Delta_{n}\right]$ in the centering term here again. So we are in the same situation as in the first part of (i), with $u_{n}=\sqrt{\Delta_{n}}$ and $\zeta_{n}=f\left(X_{\Delta_{n}} / \sqrt{\Delta_{n}}\right)$, and the only things to prove are (6.6), and also (6.5) with $\mathbb{E}\left(f(\sigma U)^{2}\right)-\mathbb{E}(f(\sigma U))^{2}$ on the right side. These two facts follow from (5.13). 
Finally, the claims in (i-2) and (ii-2) readily follow, upon using (5.12) and (5.14) (observe that the right side of $(5.12)$ is always $\mathrm{o}(\sqrt{t})$ when $r<1)$.

\subsection{Proof of Theorem 2.6}

Step 1. Here we prove (a). We denote by $\left(\mathcal{G}_{t}\right)$ the filtration on the extended space generated by $X$ and $W^{\prime}$ and all processes $U_{p} 1_{\left\{T_{p} \leq t\right\}}$. When $g$ vanishes around 0 , then $Z(g)_{t}$ is well defined because the associated sum in (2.9) is finite, and if further $F\left(g^{2}\right)<\infty$ then $Z(g)$ is clearly a locally square-integrable $\left(\mathcal{G}_{t}\right)-$ martingale whose predictable bracket is $c F\left(g^{2}\right) t$.

Now let $g \in \mathcal{E}_{1}^{\prime \prime}$. We write $g_{n}=\left(1-\psi_{1 / n}\right) g$ (recall $\left.(5.1)\right)$. By what precedes $Z\left(g_{n}\right)$ is well defined, and also

$$
\mathbb{E}\left(\left(Z\left(g_{1 / n}-g_{1 / m}\right)_{t}\right)^{2}\right)=c F\left(\left(g_{1 / n}-g_{1 / m}\right)^{2}\right) t \leq C t \int_{\{|x| \leq 2 / n\}} x^{2} F(\mathrm{~d} x)
$$

for $m \geq n$, because $|g(x)| \leq C|x|$ near 0 . Then the sequence $Z\left(g_{1}-g_{1 / n}\right)_{t}$ converges in $\mathbb{L}^{2}$ to a limit naturally denoted as $Z\left(g_{1}\right)_{t}$, and the process $Z\left(g_{1}\right)$ is a locally square-integrable $\left(\mathcal{G}_{t}\right)$-martingale. It remains to put $Z(g)=Z\left(g_{1}\right)+Z\left(g_{1}\right)$.

Step 2. Now we prove (b) when $f$ is $C^{1}$ and vanishes on the interval $[-2 \varepsilon, 2 \varepsilon]$ for some $\varepsilon>0$. We use the notation $X(\varepsilon), S_{p}, R_{p}, R_{p}^{\prime n}$ and $\Omega_{n}(T, \varepsilon)$ given around (5.3). We have $\left\{S_{p}<\infty\right\}=\cup_{q}\left\{S_{p}=T_{q}<\infty\right\}$, so if we set $U_{p}^{\prime}=U_{q}$ on the set $\left\{S_{p}=T_{q}<\infty\right\}$ the process

$$
Z_{t}^{\prime}=\sum_{p: S_{p} \leq t} \sigma f^{\prime}\left(R_{p}\right) U_{p}^{\prime}
$$

has clearly the same distribution, conditional on the $\sigma$-field $\mathcal{F}$, as $Z\left(f^{\prime}\right)$ given by (2.9). So the claim amounts to the stable convergence in law, toward $Z^{\prime}$, for the sequence of processes $Z^{n}(f)=\frac{1}{\sqrt{\Delta_{n}}}\left(V^{n}(f)-V(f)^{(n)}\right)$.

On the set $\Omega_{n}(T, \varepsilon)$ we have (5.4) for $t \leq T$, hence also

$$
Z^{n}(f)_{t}=\sum_{p: S_{p} \leq \Delta_{n}\left[t / \Delta_{n}\right]} f^{\prime}\left(R_{p}+\widetilde{R}_{p}^{\prime n}\right) \frac{R_{p}^{\prime n}}{\sqrt{\Delta_{n}}}
$$

for all $t \leq T$, where $\widetilde{R}_{p}^{n}$ is between $R_{p}$ and $R_{p}+R_{p}^{\prime n}$. Since $R_{p}^{n} \rightarrow 0$ and $\Omega_{n}(T, \varepsilon) \rightarrow \Omega$, the result will easily follow from the following property, for any integer $q \geq 1$ :

$$
\text { the random vectors }\left(R_{p}^{\prime n} / \sqrt{\Delta_{n}}\right)_{1 \leq p \leq q} \text { converge stably in law to }\left(\sigma U_{p}^{\prime}\right)_{1 \leq p \leq q} \text {. }
$$

We have $R_{p}^{\prime n} / \sqrt{\Delta_{n}}=A_{p}^{n}+A_{p}^{\prime n}$, where $A_{p}^{n}=\Delta_{i}^{n} X^{c} / \sqrt{\Delta_{n}}$ and $A_{p}^{\prime n}=\Delta_{i}^{n}\left(X(\varepsilon)-X^{c}\right) / \sqrt{\Delta_{n}}$ on the set $\left\{S_{p} \in\right.$ $\left((i-1) \Delta_{n}, i \Delta_{n}\right]$. Observe that, since $X(\varepsilon)$ is independent of $S_{p}$, the variable $A_{p}^{\prime n}$ has the same law as $(X(\varepsilon)-$ $\left.X^{c}\right)_{\Delta_{n}} / \sqrt{\Delta_{n}}$, which goes to 0 in probability by Lemma 4.1. Hence it is enough to prove $(6.8)$ when $R_{p}^{\prime n}$ is substituted with $A_{p}^{n}$.

On the other hand, the random vector $\left(A_{p}^{n}\right)_{1 \leq p \leq q}$ is distributed, conditionally on $\Omega_{n}(T, \varepsilon)$, as the vector $\left(\sigma U_{p}^{\prime}\right)_{1 \leq p \leq q}$, and it is clearly asymptotically independent of the process $X^{c}$, hence of the process $X$ as well. Then we have (6.8) and the result is proved.

Step 3. Here we prove (b) for $f$ of class $C^{1}$, and twice differentiable in a neighborhood of 0 and $f^{\prime \prime}(x)=\mathrm{o}(|x|)$ as $x \rightarrow 0$. Set $f_{\eta}=f \psi_{\eta}$. Step 2 yields that $Z^{n}\left(f-f_{\eta}\right)$ converges stably in law to $Z\left(\left(f\left(1-\psi_{\eta}\right)\right)^{\prime}\right)$ for any $\eta>0$, whereas the argument of Step 1 yields that $Z\left(\left(f\left(1-\psi_{\eta}\right)\right)^{\prime}\right) \stackrel{\text { u.c.p. }}{\longrightarrow} Z\left(f^{\prime}\right)$ as $\eta \rightarrow 0$. Then the result follows from the property

$$
\lim _{\eta \rightarrow 0} \limsup _{n} \mathbb{P}\left(\sup _{t \leq T}\left|Z^{n}\left(f_{\eta}\right)_{t}\right|>\varepsilon\right)=0, \quad \forall \varepsilon>0, \forall T>0 .
$$


We know that $Z^{n}\left(f_{\eta}\right)_{t}$ is the sum of $\left[t / \Delta_{n}\right]$ i.i.d. variables distributed as $\zeta(\eta)_{n}=\left(f_{\eta}\left(X_{\Delta_{n}}\right)-V\left(f_{\eta}\right)_{\Delta_{n}}\right) / \sqrt{\Delta_{n}}$. Furthermore, the assumptions on $f$ imply that for $\eta$ small enough then $f_{\eta}$ is $C^{2}$. Then if we look at Step 4 of the proof of Theorem 2.1-(i) we have $f_{\eta}(X)-V\left(f_{\eta}\right)=A(\eta)+M(\eta)$ where $A(\eta)$ and $M(\eta)$ are associated with $f_{\eta}$ as $A$ and $M$ are with $f$. Since $\mathbb{E}\left(M(\eta)_{t}\right)=0,(6.9)$ will obviously follow from the two properties

$$
\lim _{\eta \rightarrow 0} \limsup _{n} \Delta_{n}^{-3 / 2} \mathbb{E}\left(\left|A(\eta)_{\Delta_{n}}\right|\right)=0, \quad \lim _{\eta \rightarrow 0} \limsup _{n} \Delta_{n}^{-2} \mathbb{E}\left(M(\eta)_{\Delta_{n}}^{2}\right)=0 .
$$

Now we associate the functions $\alpha_{\eta}$ and $\alpha_{\eta}^{\prime}$ with $f_{\eta}$ by (5.5) and (5.6) (use the versions for $r=2$ in these formula, and note that $f_{\eta}^{\prime \prime}(0)=0$; note also that $g=g_{\eta}$ and $k=k_{\eta}$ depend on $\eta$ here). Recalling that $\mathbb{E}\left(\left|A(\eta)_{t}\right|\right) \leq \int_{0}^{t} H_{s}\left(\left|\alpha_{\eta}\right|\right) \mathrm{d} s$ and $\mathbb{E}\left(M(\eta)_{t}^{2}\right)=\int_{0}^{t} H_{s}\left(\alpha_{\eta}^{\prime}\right) \mathrm{d} s$, it thus remains to prove that

$$
\lim _{\eta \rightarrow 0} \limsup _{t \rightarrow 0} t^{-1 / 2} H_{t}\left(\left|\alpha_{\eta}\right|\right)=0, \quad \lim _{\eta \rightarrow 0} \limsup _{t \rightarrow 0} t^{-1} H_{t}\left(\alpha_{\eta}^{\prime}\right)=0 .
$$

The assumptions on $f$ imply that for $\eta$ small enough $\left|f_{\eta}^{\prime \prime}(x)\right| \leq \varepsilon_{\eta}(\eta \wedge|x|)$ for a family $\varepsilon_{\eta}$ of positive numbers going to 0 as $\eta \rightarrow 0$. Then one can checks that the functions $g_{\eta}$ and $k_{\eta}$ satisfy the following, where $\varepsilon_{\eta}^{\prime} \rightarrow 0$ as $\eta \rightarrow 0$ :

$$
\left|g_{\eta}(x, y)\right| \leq \varepsilon_{n}^{\prime} \phi_{2}(x) \phi_{1}(y), \quad k_{\eta}(x, y)^{2} \leq \varepsilon_{n}^{\prime} \phi_{2}(x) \phi_{2}(y) .
$$

We deduce that, for some other family $\varepsilon_{\eta}^{\prime \prime}$ still going to 0 as $\eta \rightarrow 0$, we have $\left|\alpha_{\eta}\right| \leq \varepsilon_{n}^{\prime \prime} \phi_{1}$ and $\alpha_{\eta}^{\prime} \leq \varepsilon_{n}^{\prime \prime} \phi_{2}$. Then (5.11) and (5.8) yield the first and the second parts of (6.10), respectively. Hence (b) is completely proved.

Step 4. Finally we prove (c). When $f=g$ with $g(x)=x^{2}$, this is Theorem $6.1-(\mathrm{b})$ of [8]: the process $\bar{Z}_{n}$ of this theorem is indeed $\frac{1}{2} Z^{n}\left(g^{\prime}\right)$; this theorem is proved when $\Delta_{n}=1 / n$, but the proof works equally well for any sequence $\Delta_{n} \rightarrow 0$. Moreover a close look at that proof shows that it also ensures the stable convergence in law of the pair $\left(Z^{n}(g), Z^{n}(h)\right)$ toward $\left(Z\left(g^{\prime}\right)+c \sqrt{2} W^{\prime}, Z\left(h^{\prime}\right)\right)$ (for the Skorokhod topology on the set of $\mathbb{R}^{2}$-valued càdlàg functions), as soon as $h$ is $C^{1}$ and vanishes around 0 . Then the sums $Z^{n}(g)+Z^{n}(h)$ stably converge in law to $Z\left(g^{\prime}\right)+c \sqrt{2} W^{\prime}+Z\left(h^{\prime}\right)$. Then if $f \in \mathcal{E}_{2} \cap C^{1}$ the function $h=f-g$ is as above, hence we get the result.

\subsection{Proof of Theorem 2.7}

Let us first consider the case where $J^{\prime}$ and $J^{\prime \prime}$ are empty, so we only have components of the first type. We can then reproduce the previous proof, with a function $f$ which is multidimensional with components $f_{j}$. When $f$ (that is, all $f_{j}$ 's) vanishes on a neighborhood of 0 , we have $(6.7)$ with $f^{\prime}=\left(f_{1}^{\prime}, \cdots, f_{d}^{\prime}\right)$ and the variables $\widetilde{R}_{p}^{\prime n}$ depend on $j$, but with the same $R_{p}^{\prime n}$ for all $j$. Then we deduce from (6.8) the stable convergence in law toward $Z\left(f^{\prime}\right)=\left(Z\left(f_{1}^{\prime}\right), \cdots, Z\left(f_{d}^{\prime}\right)\right)$ with the same sequence $U_{p}^{\prime}$. Next, Step 3 of the previous proof is performed component by component, hence there is nothing to change, and the result is proved.

In a second step we consider the case where $J$ is empty. Since the limit is then continuous, the conditions for convergence given in Lemmas 4.2 and 4.3 are componentwise, except for the second one in (4.3). Moreover for each component these conditions are satisfied, as we have shown in the proof of Theorem 2.5. Hence the only thing to show is the relevant extension of (6.5). To write it, we set

$$
u_{n}(j)=\left\{\begin{array}{ll}
\Delta_{n}^{1 / 2-r(j) / 2} & \text { if } j \in J^{\prime} \\
\Delta_{n}^{1 / 2} & \text { if } j \in J^{\prime \prime}
\end{array} \quad \zeta_{n}^{j}= \begin{cases}\phi_{r(j)}\left(X_{\Delta_{n}}\right) & \text { if } j \in J^{\prime} \\
f_{j}\left(X_{\Delta_{n}} / \sqrt{\Delta_{n}}\right) & \text { if } j \in J^{\prime \prime}\end{cases}\right.
$$

Then we need to show that

$$
\frac{1}{\Delta_{n}} u_{n}(j) u_{n}(k)\left(\mathbb{E}\left(\zeta_{n}^{j} \zeta_{n}^{k}\right)-\mathbb{E}\left(\zeta_{n}^{j}\right) \mathbb{E}\left(\zeta_{n}^{k}\right)\right) \rightarrow c^{\prime j k}
$$


where $c^{\prime}$ is given by (2.10). But this is a trivial consequence of (5.11), (5.13) and (5.15).

It remains to consider the general case. Let us denote $Y^{n}(1)=\left(Y^{n, j}\right)_{j \in J}$ and $\left.Y^{n}(2)=Y^{n, j}\right)_{j \in J^{\prime} \cup J^{\prime \prime}}$. These two sequences converge stably in law to their limits, say $Y(1)$ and $Y(2)$. Moreover $X^{(n)}$ (the discretized process) has the same jumps than $Y^{n}(1)$, and converges (pointwise) to $X$, and all jumps of $Y(1)$ are jumps of $X$, and $Y(2)$ is continuous: so in fact the pairs $\left(X^{(n)}, Y^{n}(1)\right)$ and $\left(X^{(n)}, Y^{n}(2)\right)$ converge stably in law to $(X, Y(1))$ and $(X, Y(2))$ respectively. Using once more the continuity of $Y(2)$ we deduce the tightness of the sequence $Z^{n}=\left(X^{(n)}, Y^{n}(1), Y^{n}(2)\right)$.

Take any subsequence $Z^{n_{k}}$ which converges in law to a limit $Z^{\prime}=\left(X^{\prime}, Y^{\prime}(1), Y^{\prime}(2)\right)$. This limit is necessarily a Lévy process, and $\left(X^{\prime}, Y^{\prime}(1)\right)$ and $\left(X^{\prime}, Y^{\prime}(2)\right)$ have (separately) the same laws as $(X, Y(1))$ and $(X, Y(2))$ : hence $X^{\prime}$ and $Y^{\prime}(2)$ are independent, and $Y^{\prime}(1)$ has no continuous Gaussian part and $Y^{\prime}(2)$ is continuous. By a well known result on multidimensional Lévy processes, this implies that $Y^{\prime}(1)$ and $Y^{\prime}(2)$ are also independent. In other words the law of $Z^{\prime}$ is the product of the laws of $(X, Y(1))$ and of $Y(2)$, and it follows that the original sequence $Z^{n}$ converges in law to the process $Z=(X, Y(1), Y(2)$ where $Y(2)$ is independent of $(X, Y(1))$.

It remains to apply once more the pointwise convergence $X^{(n)} \rightarrow X$, plus the fact that the $\sigma$-field $\mathcal{F}$ is $\sigma\left(X_{t}: t \geq 0\right)$ : we then deduce from the convergence of $Z^{n}$ to $Z$, that $Y^{n}$ converges stably in law to $Y=\left(Y(1), Y(2)\right.$ with $Y(2)$ independent of $\left(X, Y(1)\right.$ and $Y(1)=\left(Z\left(f_{f}\right)\right)_{j \in J}$, and we are done.

\section{Proofs of the theorems about $\bar{V}^{n}(f)$ And $\bar{V}^{\prime n}(f)$}

Proof of Theorem 3.2. (i,ii) It is obviously enough to prove the convergence of $Y_{t}^{n}=\frac{1}{\sqrt{T_{n}}}\left(\bar{V}^{n}(f)_{t}-H_{\Delta_{n}}(f)[n t]\right)$. Now $Y_{t}^{n}$ is the sum of $[n t]$ i.i.d. variables distributed as $\zeta_{n}-\mathbb{E}\left(\zeta_{n}\right)$, with $\zeta_{n}=f\left(X_{\Delta_{n}}\right) / \sqrt{T_{n}}$. We have $\left|\zeta_{n}\right| \leq C / \sqrt{T_{n}} \rightarrow 0$ and $\mathbb{E}\left(\zeta_{n}^{2}\right)=\Gamma_{\Delta_{n}}(f) / T_{n}$. Therefore Lemma 5.5-(a,b) implies that $n \mathbb{E}\left(\zeta_{n}^{2}\right)$ converges to $F\left(f^{2}\right)$ if $f$ is as described in (i), and to $F\left(f^{2}\right)+c\left(m_{2}-m_{1}^{2}\right)$ if $f \in \mathcal{E}_{1}^{\prime b} \cap C^{0, F}$ : the result follows from a standard CLT.

(iii) Let $f \in \mathcal{E}_{r}^{b}$ and $r<1$ and either $c>0$ or $2 r \notin I$. By Lemma 5.5-(c) we have $\Gamma_{\Delta_{n}}(f) / \Delta_{n} \rightarrow \infty$, hence a fortiori $u_{n}:=1 / \sqrt{n \Gamma_{\Delta_{n}}(f)} \rightarrow 0$, and exactly as above it is enough to prove the convergence of $Y_{t}^{n}=u_{n}\left(\bar{V}^{n}(f)_{t}-H_{\Delta_{n}}(f)[n t]\right.$, and $Y_{t}^{n}$ is the sum of $[n t]$ i.i.d. variables distributed as $\zeta_{n}-\mathbb{E}\left(\zeta_{n}\right)$, with $\zeta_{n}=u_{n} f\left(X_{\Delta_{n}}\right)$. We have $\left|\zeta_{n}\right| \leq C u_{n} \rightarrow 0$ and $\mathbb{E}\left(\zeta_{n}^{2}\right)=1 / n$, so we conclude as in (i).

Proof of Theorem 3.3. We can replace $t$ by $[n t] / n$ in the centering term of $Y^{n}$. Then the proof of the first claim goes exactly as for Theorem 2.5, except that we use the usual $q$-dimensional CLT and we do not need something like (6.6). For the last claim, we use (5.12) and (5.14).

Proof of Theorem 3.1. (i) In all cases we deduce from Theorem 3.2-(i,ii) that

$$
\frac{1}{T_{n}}\left(\bar{V}^{n}(f)_{t}-n H_{\Delta_{n}}(f) t\right)=\frac{1}{T_{n}} \bar{V}^{n}(f)_{t}-\frac{t}{\Delta_{n}} H_{\Delta_{n}}(f) \stackrel{\text { u.c.p. }}{\longrightarrow} 0 .
$$

Then (5.8) immediately gives the result.

(ii) and (iii): the same type of arguments, based on Theorem 3.3 and (5.11) and (5.13), gives the result.

Acknowledgements. The author whish to express his thanks to a referee who pointed out a serious mistake in a first version of this paper and helped to improve the presentation.

\section{REFERENCES}

[1] Y. Aït-Sahalia and J. Jacod, Volatility estimators for discretely sampled Lévy processes. To appear in Annals of Statistics (2005).

[2] T.G. Andersen, T. Bollerslev and F.X. Diebold, Parametric and nonparametric measurement of volatility, in Handbook of Financial Econometrics, Y. Aït-Sahalia and L.P. Hansen Eds., Amsterdam: North Holland. Forthcoming (2005). 
[3] O.E. Barndorff-Nielsen and N. Shephard, Realised power variation and stochastic volatility. Bernoulli 9 (2003) $243-265$. Correction published in pages 1109-1111.

[4] O.E. Barndorff-Nielsen, S.E. Graversen, J. Jacod, M. Podolskij and N. Shephard, A central limit theorem for realised bipower variations of continuous semimartingales, in From Stochastic calculus to mathematical finance, the Shiryaev Festschrift, Y. Kabanov, R. Liptser, J. Stoyanov Eds., Springer-Verlag, Berlin (2006) 33-69.

[5] O.E. Barndorff-Nielsen, N. Shephard and M. Winkel, Limit theorems for multipower variation in the presence of jumps. Stoch. Processes Appl. 116 (2006) 796-806.

[6] A.N. Borodin and I.A. Ibragimov, Limit Theorems for Functionals of Random Walks. Proceedings Staklov Inst. Math. 195, A.M.S. (1995)

[7] J. Jacod and A. Shiryaev, Limit Theorems for Stochastic Processes. 2nd ed., Springer-Verlag, Berlin (2003).

[8] J. Jacod and P. Protter, Asymptotic error distributions for the Euler method for stochastic differential equations. Ann. Probab. 26 (1998) 267-307.

[9] J. Jacod, The Euler scheme for Lévy driven stochastic differential equations: limit theorems. Ann. Probab. 32 (2004) $1830-1972$.

[10] J. Jacod, A. Jakubowski and J. Mémin, On asymptotic error in discretization of processes. Ann. Probab. 31 (2003) 592-608.

[11] D. Lépingle, La variation d'ordre $p$ des semimartingales. Z. für Wahr. Th. 36 (1976) 285-316.

[12] C. Mancini, Disentangling the jumps of the diffusion in a geometric jumping Brownian motion. Giornale dell'Instituto Italiano degli Attuari LXIV (2001) 19-47.

[13] J. Woerner, Power and multipower variation: inference for high frequency data, in Stochastic Finance, A.N. Shiryaev, M. do Rosário Grosshino, P. Oliviera, M. Esquivel Eds., Springer-Verlag, Berlin (2006) 343-354. 